\title{
Improvisation et travail ordinaire des enseignants entrant dans le métier. Quelle activité ? Quels enjeux?
}

The improvisation and ordinary work of new teachers. Which activity?

What challenges?

Guillaume Azéma

\section{(2) OpenEdition}

Journals

Édition électronique

URL : http://journals.openedition.org/activites/3941

DOI : 10.4000/activites.3941

ISSN : 1765-2723

Éditeur

ARPACT - Association Recherches et Pratiques sur les ACTivités

Référence électronique

Guillaume Azéma, « Improvisation et travail ordinaire des enseignants entrant dans le métier.

Quelle activité ? Quels enjeux ? », Activités [En ligne], 16-1 | 2019, mis en ligne le 15 avril 2019, consulté le 09 août 2019. URL : http://journals.openedition.org/activites/3941 ; DOI : 10.4000/activites.3941

Ce document a été généré automatiquement le 9 août 2019

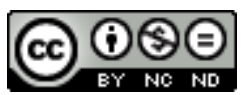

Activités est mis à disposition selon les termes de la licence Creative Commons Attribution - Pas d'Utilisation Commerciale - Pas de Modification 4.0 International. 


\section{Improvisation et travail ordinaire des enseignants entrant dans le métier. Quelle activité ? Quels enjeux?}

The improvisation and ordinary work of new teachers. Which activity?

What challenges?

Guillaume Azéma

\section{NOTE DE L'ÉDITEUR}

Article soumis le 10 février 2018, accepté le 10 février 2019

\section{Introduction}

1 Improvisation. À l'école, elle est l'un de ces mots qu'utilisent parfois les gens de métier. Un de ces termes qui circulent, sans avoir pignon sur rue, pour dire une forme de réalité $\mathrm{du}$ travail, peut-être inavouable. On la rencontre encore, cette fois haut et fort, pour caractériser et repousser l'amateurisme douteux, ou chanter les louanges des experts ou des virtuoses. Les textes institutionnels souvent la taisent ${ }^{1}$. D'emblée, le caractère disparate des degrés de «désirabilité collective», donc de «légitimité culturelle » (Lahire, 2006) de cette pratique, interroge.

Depuis plus de 40 ans, dans le champ des recherches en sciences de l'éducation et en didactique, la notion d'improvisation est utilisée: pour décrire et comprendre les interactions en classe (e.g. Erickson, 1982 ; Gershon, 2006 ; Sawyer, 2004), pour proposer un éclairage sur la façon dont, dans le cours de leurs dynamiques, apprennent les élèves ( e.g. Baker-Sennett \& Matusov, 1997), enseignent les professeurs (e.g. Borko \& Livingstone, 
1989, Perrenoud, 1994; Tochon, 1993), mais aussi pour étudier et transformer la formation de ces derniers (e.g. Pelletier \& Jutras, 2008), ou encore pour parler de situations d'apprentissage spécifiques et analyser leur portée (e.g. Gagnon, 2011). On comprend que le terme participe de diverses compréhensions, qu'il peut recouvrir différents phénomènes, des significations plurielles et des points de vue plus ou moins contradictoires. Nous verrons que les façons dont il est considéré ne sont pas sans déboucher sur certains hiatus et que le cheminement consistant à penser à partir de ces hiatus nous semble propre à alimenter une herméneutique de la complexité du travail enseignant.

3 Dans le champ des sciences de l'éducation, notre recherche prend acte de l'intérêt qu'il y a, suivant différents objets et approches, d'une part à continuer de comprendre les interactions en classe (particulièrement complexes et diversifiées) et les phénomènes d'enseignement-apprentissage qui potentiellement, pour tous les acteurs qui y participent, y ont cours; et d'autre part à continuer de décrire et de comprendre les dynamiques ayant possiblement à voir avec la (ré)génération des acteurs de l'école notamment dans un contexte marqué par le caractère problématique, sur le plan de leur fécondité, des logiques contradictoires dont fait l'objet le travail enseignant, entre initiatives et contrôle, professionnalisation et déprofessionnalisation (Maroy \& Cattonar, 2002 ; Zoïa \& Visier, 2013).

4 Le présent article est le fruit d'une recherche au cours de laquelle nous nous sommes attachés à analyser l'activité qui, pour les enseignants entrant dans le métier ${ }^{2}$, porte le nom d'improvisation. Notre travail nous a conduits à prendre nos distances avec le dualisme classique de l'improvisation, entre "valorisation mythique " (la pratique virtuose des rares experts d'une profession, devenue quasi magique suite à des années de labeur) et "dévalorisation critique " (la pratique approximative, voire inopérante, des incompétents, ou de professionnels en devenir), mis au jour par De Raymond (1980), pour approcher certaines particularités et subtilités du rapport entre travail enseignant et improvisation.

5 Les données ont été construites et analysées dans le cadre d'une anthropologie culturelle enactive (Theureau, 2015) - donc selon une méthode associant ethnographie et analyse de l'activité, construction et analyse de données extrinsèques et intrinsèques, tout en respectant le principe de primat de l'intrinsèque (primat au point de vue des acteurs enquêtés). Conduite sur de longs mois, elle a fait de l'improvisation à la fois un objet et un instrument de connaissance - autrement dit, en tous les cas, une clé de compréhension.

La structure générale de l'article s'organise comme suit. Dans un premier temps, nous proposons un état de l'art permettant de poser une série de problèmes concernant les rapprochements entre improvisation et enseignement-apprentissage scolaire. Cet état de l'art ouvre sur la définition de notre objet de recherche, associé au cadre théorique de l'étude. Nous précisons ensuite le cadre méthodologique de la construction et de l'analyse des données. Nous poursuivons par une présentation des principaux résultats. Ils débouchent sur une façon particulière de considérer l'improvisation et ses caractéristiques dans le cas de l'activité des néo-enseignants. S'en suit une discussion comprenant des réflexions qui concernent le travail enseignant, le développement professionnel et la formation à ce travail. 


\section{1. État de l'art et construction de problèmes}

7 Pour la recherche dans son ensemble, notre état de l'art s'est attaché à faire un point sur la question de l'improvisation en enseignement-apprentissage scolaire. D'une revue de littérature internationale (Azéma, 2015), émergent pour nous 3 principaux problèmes dignes d'intérêt : a) la notion fait l'objet de diverses conceptions dont nous considérons que certaines affaiblissent son potentiel herméneutique, b) en son sens louable, elle caractérise d'abord et pour la plupart des auteurs, les interactions des enseignants experts ou chevronnés, c) elle est prioritairement pensée en lien avec l'apprentissagedéveloppement des élèves. Ces problèmes ont contribué à la circonscription de notre objet de recherche.

\subsection{La notion d'improvisation : définitions et points de vigilance}

8 Dans les recherches concernant le travail enseignant et l'apprentissage scolaire, l'improvisation apparaît comme une notion polysémique. Si les sens qui lui sont attribués participent souvent d'une herméneutique féconde, ils peuvent aussi perturber ou interdire des compréhensions. Nous pointons ci-après certaines limites, qui justifient selon nous de reprendre les investigations selon un autre angle :

a. Dans les conceptions radicalement microsociologiques ou socio-culturalistes (e.g. Erickson, 1982; Sawyer, 2004), il est possible de considérer que les interactions humaines, dans leur ensemble, sont improvisation. Cette confusion, pour pertinente qu'elle soit d'un certain point de vue, présente corrélativement le risque d'un affaiblissement de son pouvoir herméneutique concernant des classes particulières d'interactions. En effet, si toutes les interactions humaines sont improvisation alors, dans le cours d'une interaction, rien ne l'est en particulier. Or, il nous parait important d'insister sur le fait que, même si l'on considère que toute interaction humaine est plus ou moins improvisée, il peut être intéressant de comprendre ce qui fait le «plus» ou le «moins». Il faut noter que dans cette difficulté à définir et à différencier ce qui est plus ou moins improvisationnel (ou ce qui est improvisation de ce qui ne l'est pas), les acteurs des interactions étudiées ne sont pas directement sollicités pour pointer ce qui, pour eux, est de l'ordre de l'improvisation (e.g. Erickson, 1982 ou Gershon, 2006);

b. Certaines recherches en font une notion très proche, voire confondue avec celle d'adaptation (e.g. Tochon, 1993). À trop forcer le rapprochement entre improvisation et adaptation, nous pensons que l'on perd en précision herméneutique concernant le travail enseignant. Si l'on peut considérer que l'improvisation comprend de l'adaptation, il nous semble qu'elle ne se confond pas avec elle. À l'instar de Sartre (2005, p. 31) concernant le concept d'image, on peut en effet se dire que «puisque nous pouvons parler [d'improvisation], puisque ce terme a un sens pour nous - il faut que [l'improvisation], prise en elle-même, renferme dans sa nature intime un élément de distinction radicale.». Le concept d'improvisation (du latin in-pro-videre: sans voir avant) semble avoir émergé pour dire le fait «de faire en l'absence de», contrairement à la notion d'adaptation. Certains auteurs insistent sur le fait qu'il ne s'agit pas de deux activités identiques et marquent leurs différences (e.g. Brown \& Edelson, 2003 ou Piot, 2008) ${ }^{3}$.

Confondre adaptation et improvisation c'est encore, potentiellement, prendre le risque de réduire l'improvisation à une réponse à une occurrence perturbatrice afin de limiter l'écart entre ce qui se passe et le but recherché par un acteur, et ce à partir d'un « geste professionnel » (Bucheton \& Soulé, 2009) stabilisé. À l'extrême, on pourrait considérer 
que cela sous-entend de faire de l'enseignant une sorte d'automate qui ne peut, comme le souligne Simondon (2013, p. 161) :

«Que s'adapter en réduisant de plus en plus l'écart qui existe entre son action et le but prédéterminé (...) [En tant qu'automate,] il n'invente pas et ne découvre pas des buts au cours de son action, car il ne réalise aucune véritable transduction... »

Peut-être serait-il intéressant d'asseoir dans le champ de l'enseignement ${ }^{4}$, une différence entre adaptation et improvisation. Par exemple, en prenant en compte la différence que font certains auteurs entre situations où les schèmes (ou les matrices cognitivopragmatiques ou les connaissances) disponibles sont suffisants pour faire avec l'imprévu, et situations où les schèmes, en l'état, ne suffisent pas (e.g. Perrenoud, 1994, suivant Piaget, et Bourdieu ; Piot, 2008 ; ou Pelletier \& Jutras, 2008), l'innovation devenant alors un second critère crucial.

\subsection{L'improvisation chez les enseignants est-elle une question d'ancienneté?}

11 La plupart du temps, dans les différents textes, il semble que la capacité à improviser efficacement ne peut être que l'apanage des interactions concernant les enseignants chevronnés, expérimentés (souvent confondus avec les enseignants experts). L'enseignant compétent, le "bon enseignant " sait improviser ou participer à une improvisation (e.g. Erickson, 1982 ; Lobman, 2003 ; Sawyer, 2004 ; Yinger, 1987a), ou aime improviser (Lopes de Maturana Luna, 2010). Il est aussi considéré que l'habileté improvisationnelle caractérise l'expert (Borko \& Livingston, 1989; Tochon, 1993) qui " "jongle" avec les routines et peut improviser à partir du matériau intériorisé en un arrangement constamment renouvelé » (Tochon, 1992, p. 186).

12 Ce point de vue, dont nous nous demandons s'il ne fait pas l'objet d'une forme de

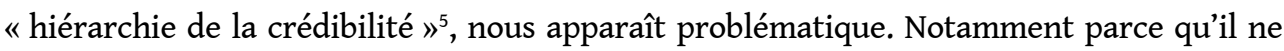
permet que difficilement de donner une valeur à la pratique des enseignants novices qui, dès lors, est d'abord appréhendée en creux (en termes de manques, d'insuffisances...) en référence à celle des enseignants chevronnés (Borko \& Livingston, 1989 ; Erickson, 2011 ; Tochon, 1993 ; Yinger, 1987b).

13 Remarquons que la moindre aptitude à improviser, pointée dans les textes pour ce qui concerne les enseignants novices, et pensée en termes de manques ou d'insuffisance, pourrait être appréhendée en rapport : a) à un défaut d'autorisation des novices, qui s'interdisent, plus ou moins consciemment, une praxis que la culture professionnelle réprouve à leur niveau, et b) à un engoncement dans un imbroglio d'injonctions qui valorisent l'élaboration et le respect de plans programmes (Suchman, 1990). De son côté DeZutter (2011) note la valeur supérieure donnée, explicitement et implicitement, à la planification. Fournier (2011) souligne que les programmes de formation d'enseignants sont largement structurés selon une conception rationaliste de l'enseignement et qu'ils ne proposent que rarement un entraînement en improvisation pédagogique. Pour Lobman et Lundquist (2007), les enseignants sont plus souvent entraînés à ne pas improviser.

14 Notons que des auteurs se décalent du point de vue majoritaire et participent à sa mise en débat. Kelly, Brown et Crawford (2000) pondèrent la conception selon laquelle il faut à l'enseignant un bagage important de connaissances disciplinaires pour improviser efficacement au service de la construction de compétences par les élèves. Shem-Tov 
(2011) montre comment, en situation d'enseignement à l'école primaire, une étudiante de troisième année au professorat des Arts et de la danse improvise avec une efficience remarquable dans deux situations potentiellement problématiques (en utilisant les débordements d'élèves au profit des apprentissages en cours). Définissant l'improvisation comme une « invention en situation inédite ", Piot (2008) prend le contre-pied du point de vue classique d'une aisance supérieure à improviser chez les enseignants chevronnés. Il considère que ces derniers sont souvent déstabilisés par l'inédit et les pense plutôt particulièrement performants en adaptation (i.e. en ajustement au contexte, sur la base d'une riche " matrice cognitivo-pragmatique »). Pour lui, l'improvisation est en revanche incontournable dans la pratique des néo-enseignants. Pour Baker-Sennett et Matusov (1997), la question de savoir si l'improvisation bénéficie des structures et de l'expérience n'a jamais été minutieusement examinée. La différence entre les enseignants est d'abord liée à un changement de paradigme impliquant : a) un abandon du contrôle du processus éducationnel, et b) la considération de l'enseignement-apprentissage comme un effort collaboratif. Ils citent Johnstone qui précise que «les mauvais improvisateurs bloquent l'action, souvent avec un haut niveau de compétences. Les bons improvisateurs permettent à l'action de se développer " (Ibid. p. 199). Il n'est pas dit que les jeunes enseignants en sont incapables.

\subsection{L'improvisation en interaction en classe, prioritairement au service de l'apprentissage-développement des élèves}

En résonnance avec le point précédent, nous nous interrogeons sur combien il semble aisé pour les auteurs, de défendre le caractère incontournable de l'improvisation pour ce qui concerne l'apprentissage-développement des élèves (e.g. Baker-Sennett \& Matusov, 1997) - l'improvisation est alors plutôt conçue comme un faire sans voir, ni savoir avant, comme le processus même de construction, de création du savoir, à partir du bagage disponible, et sur la base de tentatives, de bricolage, d'expérimentation, etc., individuelles ou, plus souvent, collectives. La chose est davantage sous-entendue côté enseignant, pour lequel il est plus clairement attendu qu'il soit déjà savant sur le plan improvisationnel ${ }^{6}$ ( e.g. Sawyer, 2011). C'est donc sous sa forme aboutie, maitrisée voire virtuose et en relation à une large expérience qu'elle est d'abord mise en avant. Il est en effet majoritairement considéré qu'il faut être professionnel pour improviser juste, en classe, et rares sont les études qui s'interrogent sur la proposition inverse, à savoir : il faut improviser, en classe, pour devenir professionnel (ce qui semble être le point de vue de Piot, 2008 ou de Pelletier \& Jutras, 2008). Le développement de la compétence en improvisation est le plus souvent relégué à des situations spécifiques de formation hors la classe.

Pourtant, si l'on considère qu'improviser c'est apprendre et se développer, dans une forme de co-création (Sawyer, 2004), cela doit valoir, au cours des interactions en classe, à la fois pour les élèves et pour les enseignants. En fait, si de façon récurrente, les recherches ont très clairement mis en évidence l'illusion d'une pure liberté de l'improvisation en enseignement scolaire - insistant sur son caractère "disciplined" (Sawyer, 2004, 2011), « réglé » (Perrenoud, 1994), elles sont généralement beaucoup plus laconiques sur le pouvoir que peut avoir l'improvisation, de transformer/dépasser ce qui la règle ou la discipline. Même si pour certains chercheurs, il semble entendu que le background de l'improvisation (routines, schémas, modèles contextuels de pensée et d'action, etc.) se crée en improvisant (e.g. Erickson, 2011), par la pratique (Yinger, 1987a). 

l'improvisation dans le travail des enseignants entrant dans le métier. D'autant que, dans le cours d'une prise de fonction qui peut être « sauvage » (Huberman, 1993), et «malgré une entrée brutale dans le vif du sujet » (Ria, 2014), ces « jeunes enseignants », dans le feu des interactions en classe, parviennent à s'en sortir à coup de "passages à risque ", d'« arrangements astucieux» (Ria, 2012), en faisant avec une pluralité de "ressources » (Perez-Roux \& Lanéelle, 2015). Continuer de comprendre le travail des néo-enseignants c'était, potentiellement, se donner les moyens de participer à un effort consistant à soutenir ce travail, pour ce qu'il est et pour ce qu'il peut être.

\section{Objet de recherche et cadre théorique}

Pour tenter de produire de nouveaux éclairages, nous avons emboîté le pas des recherches s'inscrivant dans la dynamique des «nouveaux regards sur le travail enseignant" (Lantheaume, 2008). Suite à la bascule épistémologique à laquelle ont consisté, en recherche en éducation, le développement des approches interactionnistes subjectivistes (Gauthier, Desbiens, Malo, Martineau, \& Simard, 1997) et le «passage de la professionnalisation à l'activité » (Lantheaume, 2008), notre recherche s'inscrit dans une « approche activité » du travail enseignant.

19 Nous nous sommes demandés : ce qu'est et en quoi consiste l'activité en interaction en classe qui, pour les néo-enseignants, porte le nom d'improvisation. Nous ne sommes donc pas partis d'une définition a priori de la notion, mais avons mobilisé le mot et construit son sens pour et avec les acteurs, en approchant leur culture, et suivant une analyse de leur activité pour des moments d'interaction qu'ils associaient à de l'improvisation.

Nous prenons le terme activité dans le sens proposé par le programme de recherche «cours d'action» et suivant l'anthropologie culturelle qu'il propose, dite enactive (Theureau, 2015). Considérée comme cognitive, autonome, incarnée, située, individuelle et collective, techniquement constituée, cultivée et vécue (Theureau, 2006), elle est, suivant l'hypothèse ontologique de l'enaction, conçue comme la dynamique des interactions asymétriques entre un acteur et son environnement (Theureau, 2015). Il est par ailleurs considéré : a) que cette dynamique d'interactions asymétriques (ou activité) est accompagnée de conscience préréflexive - entendue comme ce qu'est en mesure d'en montrer, mimer, simuler, commenter, raconter l'acteur, à tout instant de son déroulement; et b) qu'elle est contrainte par (et qu'elle a des effets dans) le corps, la situation et la culture ${ }^{7}$ de l'acteur. S'en suivent des implications méthodologiques.

\section{Cadre méthodologique de la construction et de l'analyse des données}

\subsection{Un assemblage particulier}

21 En cohérence avec les fondements théoriques du programme de recherche retenu, décrire et comprendre l'activité qui pour les enseignants entrant dans le métier porte le nom d'improvisation ont impliqué un assemblage de méthodes.

Nous avons d'abord approché notre objet dans le cadre de notre mission de formateur de professeurees des écoles stagiaires, à l'occasion de visites formatives ou d'entretiens 
opportunistes. Par la suite, nous avons conduit une série systématique de 23 entretiens ethnographiques avec des enseignante's stagiaires et titulaires première année, travaillant à l'école, au collège ou à l'université. Avec certains d'entre eux/elles (volontaires et suffisamment représentatifs de notre population), nous avons réalisé une série d'observations participantes en classe. Ces observations ont pu comprendre : a) la prise de notes ethnographiques, b) la tenue d'un journal par le chercheur, c) des filmages d'interactions en classe (elles ont parfois été accompagnées d'autre temps de présence chercheur/acteur - sur son lieu de travail, lors de repas pris ensemble ou d'un hébergement chez lui - qui ont été l'occasion de nouvelles observations et d'entretiens informels) $)^{8}$. Afin de comprendre quelles interactions en classe portaient, pour les enseignants entrant dans le métier, le nom d'improvisation, nous avons construit une échelle de Likert leur permettant de caractériser de leurs interactions en classe entre $(-3)$ Estime très fortement ne pas improviser ou vivre une improvisation... $(-2,-1,0,+1,+2) \ldots$ et $(+3)$ Estime très fortement improviser ou vivre une improvisation. Nous l'avons utilisée en relation aux illustrations que les acteurs ont convoquées lors des entretiens ethnographiques, ou dans la foulée de temps d'observation directe. Enfin, suivant le passage psychophénoménologique obligé que constitue, dans le cadre du programme de recherche "cours d'action », l'appel de la conscience préréflexive, nous avons procédé à une série d'entretiens de remise en situation dynamique à partir de traces d'activité ou d'entretiens d'autoconfrontation (Theureau, 2010), sur la base de la sélection, par l'acteur, de moments pour lesquels il estimait fortement ou très fortement improviser ou vivre une improvisation $(+2$ ou +3 sur notre échelle de Likert). (Pour une présentation davantage détaillée de l'ensemble de la méthode, voir annexe 1 ).

L'ensemble de l'assemblage méthodologique mis en œuvre visait : a) un accès à la culture des acteurs, notamment concernant l'improvisation et en relation à leur travail ordinaire, b) une considération des contraintes de l'activité dans les corps des acteurs, leurs situations et leurs cultures, c) un respect du caractère situé de leur activité et de leur point de vue concernant cette dernière. Et ainsi, garantir au mieux (possible) des analyses de "cours d'action " ayant à voir avec l'improvisation telle que signifiante pour les enseignants entrant dans le métier, et telle qu'actée par eux/elles.

Les analyses réalisées sont de trois ordres: a) les entretiens ethnographiques ont fait l'objet d'une analyse de contenu thématique et sémantique, b) les entretiens de remise en situation dynamique ou d'autoconfrontation ont fait l'objet d'une analyse en signes (Durand \& Veyrunes, 2005 ; Theureau, 2006), suivie d'une analyse inductive, c) l'ensemble des données ethnographiques a permis la production de résultats sur la base du jeu de proximité/distance entre l'ethnographe et les acteurs dont il cherchait à comprendre une part de la réalité.

25 Afin de faciliter au lecteur l'accès au sens des tableaux illustratifs (ou extraits de tableaux) présentés dans la partie résultats de l'article, nous rappelons que la possibilité d'analyser en signes le couplage asymétrique acteur/environnement est chaînée à l'hypothèse de connaissance dite de l'activité-signe (ou semiosis ou production-réception de signes). Selon cette hypothèse, l'homme pense et agit par signes et la dynamique des interactions asymétriques entre un acteur et son environnement est analysable en une série de signes. Chaque signe a la même structure complexe, constituée d'un ensemble de pôles. Son organisation s'inspire des trois catégories phanéroscopiques de Peirce :

- la priméité : catégorie du possible, celle du potentiel ;

- la secondéité : catégorie de l'actuel, celle de l'occurrence, de l'action ; 
- la tiercéité : catégorie du virtuel, celle de la « loi » ou de l'habitude.

Dans le cours de son couplage asymétrique à son environnement, il est considéré que : ce qui est actuel, c'est-à-dire ce qui se passe pour/par un acteur ou ce qu'il ou elle agit en termes de sensations, sentiments ou émotions, imagination, discours privé, action, communication, etc. (pôle Unité de cours d'action de l'acteur U) ; s'actualise sur fond de ce qui fait signe ou d'une perception active qui « perturbe » l'acteur (pôle Representamen R); et sur fond de possibles pour l'acteur, c'est-à-dire sur fond: d'un savoir propre typique relativement pérenne ou habitudes" (pôle Référentiel de l'acteur $\mathbf{S}$ ), de l'anticipation d'un futur possible (pôle Attente A), et de préoccupations ou d'intentions d'arrière-plan (pôle Engagement de l'acteur E).

L'actuel est par ailleurs l'arrière-plan d'un processus « virtuel » de typicalisation. C'est à dire de découverte, création, renforcement, affaiblissement, abandon, etc. de types de savoirs ou habitudes (pôle Interprétant I). Autrement dit, d'apprentissage-développement de l'acteur.

C'est l'ensemble de ces pôles qui, dans le cadre théorique sémiologique du programme de recherche "cours d'action", constitue un signe. Une partie du signe est visible ou audible... l'autre est invisible, silencieuse (voir le schéma 2, ci-après)... mais elle est, on l'a $\mathrm{vu}$, considérée comme montrable, racontable, commentable (en partie). Chaque signe est considéré comme concaténé ou enchâssé à des signes en amont et en aval dans une dynamique temporelle complexe (s'il est concaténé avec des signes juste avant et juste après, il peut aussi l'être avec des signes qui sont plus ou moins éloignés de lui). Ce, dans un processus global continu. (Pour l'illustration d'un enchaînement de signes, voir l'annexe 2).

Figure 1 : Un signe, avec, à l'instar de l'iceberg, sa partie visible et sa partie non directement visible.

Figure 1: A sign with, like the iceberg, its visible part and its part that is not directly visible

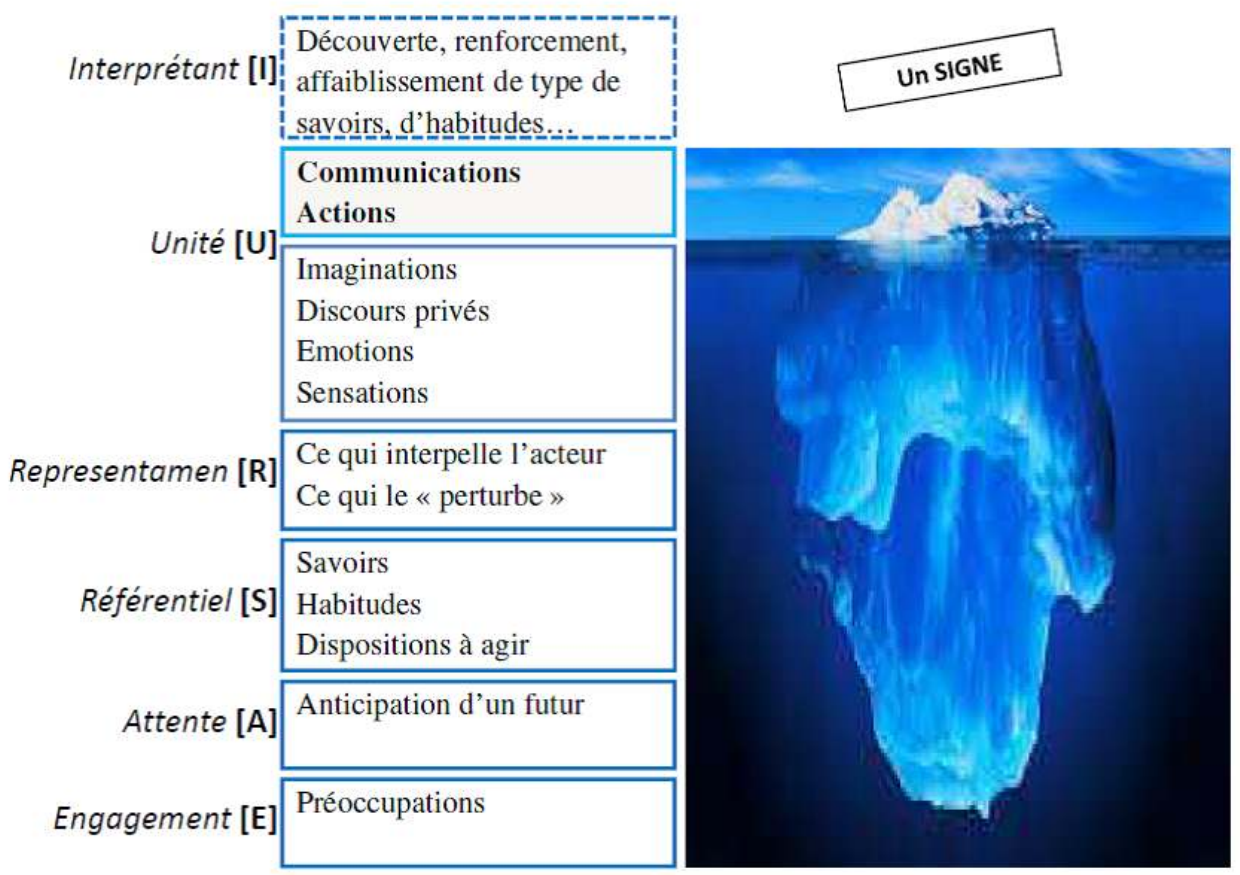




\subsection{Ethnographie et anthropologie culturelle enactive - Précisions}

'histoire des acteurs, leur contexte général de travail (tel qu'observé et tel que compris à partir des actions de l'acteur ou de ce qu'il peut en dire), leur culture, leurs préoccupations fondamentales ou thêmata (Theureau, 2015, suivant Holton), etc., permet en outre :

- un affinage de la qualité des entretiens d'autoconfrontations ou de «remise en situation dynamique » (notamment favorisant une « écoute cultivée et attentive » du chercheur, qui peut effectuer des relances en fonction de ce qu'il appréhende de l'activité des acteurs);

- de davantage assurer certaines «rétrodictions " (Theureau, 2006; Perrin, Theureau, Menu, \& Durand, 2011), c'est-à-dire à certaines inférences permettant de combler, lors des analyses, des manques dans les données construites sur la base de la seule expression de la conscience préréflexive (non seulement la conscience préréflexive ne dit pas tout, mais son expression est partielle en ce sens que l'acteur ne saurait la montrer, mimer, simuler, commenter, raconter toute, au fur et à mesure de sa dynamique qui accompagne le flux de l'activité). La rétrodiction se base sur: a) les habitudes de l'acteur, b) les événements similaires qui sont identifiés en amont ou aval du moment étudié, c) les traces de l'activité. Ceci permet de compléter la documentation de l'activité qui est prioritairement basée sur les commentaires faits par l'acteur en entretien d'autoconfrontation (Ibid. p. 170).

\section{Résultats}

Afin d'approcher l'activité qui, pour les néo-enseignants, porte le nom d'improvisation, une première série de résultats a été le fruit d'une analyse de contenu thématique et 
sémantique des entretiens ethnographiques. Nous avons entre autres pu mettre au jour ce que n'était pas l'improvisation. Dans le cadre de ce chapitre, nous commençons la présentation par ces résultats, qui sont informatifs en négatif (au sens photographique). S'en suivront ceux issus de l'analyse de "cours d'action ». Enfin nous proposerons une série de résultats produits sur la base du jeu de proximité/distance entre l'ethnographe et les acteurs dont il a tenté de comprendre l'activité-improvisation.

\subsection{Ce que n'est pas ou pas exactement l'activité-improvisation}

Ce n'est pas un «faire sans préparation ». Au sens de faire sans préparer le cours ou s'y préparer. Les néo-enseignants ne démarrent pas un cours sans avoir, dans la phase préactive, préparé quelque chose. Ils y passent un temps plus ou moins long, en des moments et des lieux divers (dans leur bureau, au cours du petit déjeuner ou de leur trajet et/ou arrivé sur le lieu de travail, etc.). Pour aller faire cours, il faut être suffisamment en confiance. À défaut d'une aisance dans l'interaction humaine, qui n'est pas pour eux gagnée d'avance, les néo-enseignants ont besoin de se sentir à l'aise avec le contenu et/ou avec l'espace de travail et/ou avec le matériel.

Elle n'est pas corrélative d'une situation où le néo-enseignant perd complètement pied, où, envahi, submergé, sous une trop forte pression émotionnelle, rien ne vient. Pour qu'il y ait sentiment d'improviser, il faut que dans la dynamique de l'interaction viennent des choses, même si c'est a minima. Muriel : «quand je suis perturbée comme ça j'improvise plus (... ) j'arrive plus à imaginer ».

37 L'activité-improvisation n'est pas corrélative d'une partie magistrale de cours qui est en parfaite adéquation avec le détail de la prévision (surtout quand cette dernière est écrite). Certains néo-enseignants parlent de «minimum syndical », d'autres de " cours froid » auquel est associée une « impression d'avoir mal fait mon métier alors que pourtant, sur le papier on a dit tout ce qu'il fallait dire " (Sébastien).

38 L'activité-improvisation n'est pas une activité sans bagage. Les néo-enseignants font régulièrement référence à un "déjà-là » tous azimuts, qui vient parfois de très loin. Flora: "C'est un truc que j'avais vu dans une vidéo à l'IUFM » (l'enseignante stagiaire fait référence «à un truc » mis en œuvre en grande section de maternelle, lors de l'entrée des élèves en classe. Marie, quant à elle, nous présente sa façon de fonctionner avec des élèves (dit difficiles) de son collège. Elle réinvestit une part d'un savoir construit au cours de 18 années de pratique de l'équitation. Il apparaît que l'efficience d'action se joue dans la rencontre fructueuse entre le potentiel du déjà-là et de celui de la situation.

L'activité-improvisation n'est pas majoritairement confondue avec l'adaptation. En tout cas, lorsque les néo-enseignants associent cette dernière à la mise en œuvre, dans le cas d'un imprévu finalement peu surprenant, de façons de faire stabilisées qui donnent, dans des conditions récurrentes et sans relief émotionnel particulier, ce qui était globalement attendu. En rapport à une séance d'EPS en maternelle Jérémy souligne qu'il a fait de petites mises en contexte improvisées : «Mais je ne suis pas allé chercher bien loin. C'était plus de l'adaptation que de l'improvisation ». Nous lui demandons la différence qu'il fait entre les deux. Il nous répond "l'adaptation c'est des choses qui arrivent régulièrement. On sait, on sent que ça va arriver (...) Ce sont de petites choses simples à régler. C'est que du réglage. Le bouton n'est pas sur la bonne fréquence, je tourne, mais je change pas de bouton ». 
40 Concernant les entretiens ethnographiques, nous avons, entre autres, calculé la fréquence des occurrences thématiques. Si l'on attribue la valeur 1 dans le cas où un acteur fait le rapprochement entre improvisation et adaptation, et la valeur 0 dans le cas contraire. On obtient une moyenne de $0,35 / 1^{11}$.

\subsection{Résultats issus de l'analyse de « cours d'action »}

41 Nous proposons ici une série de résultats concernant 4 études de cas (pour une présentation succincte des cas, voir le tableau 1, ci-après). Chacun de ces cas correspond à un moment qui a été (à chaud) fortement considéré, par chacun des acteurs, comme une improvisation (attribution d'une valeur de +2 ou +3 sur notre échelle de Likert).

Tableau 1 : Présentation succincte des cas.

Table 1 : Brief presentation of cases

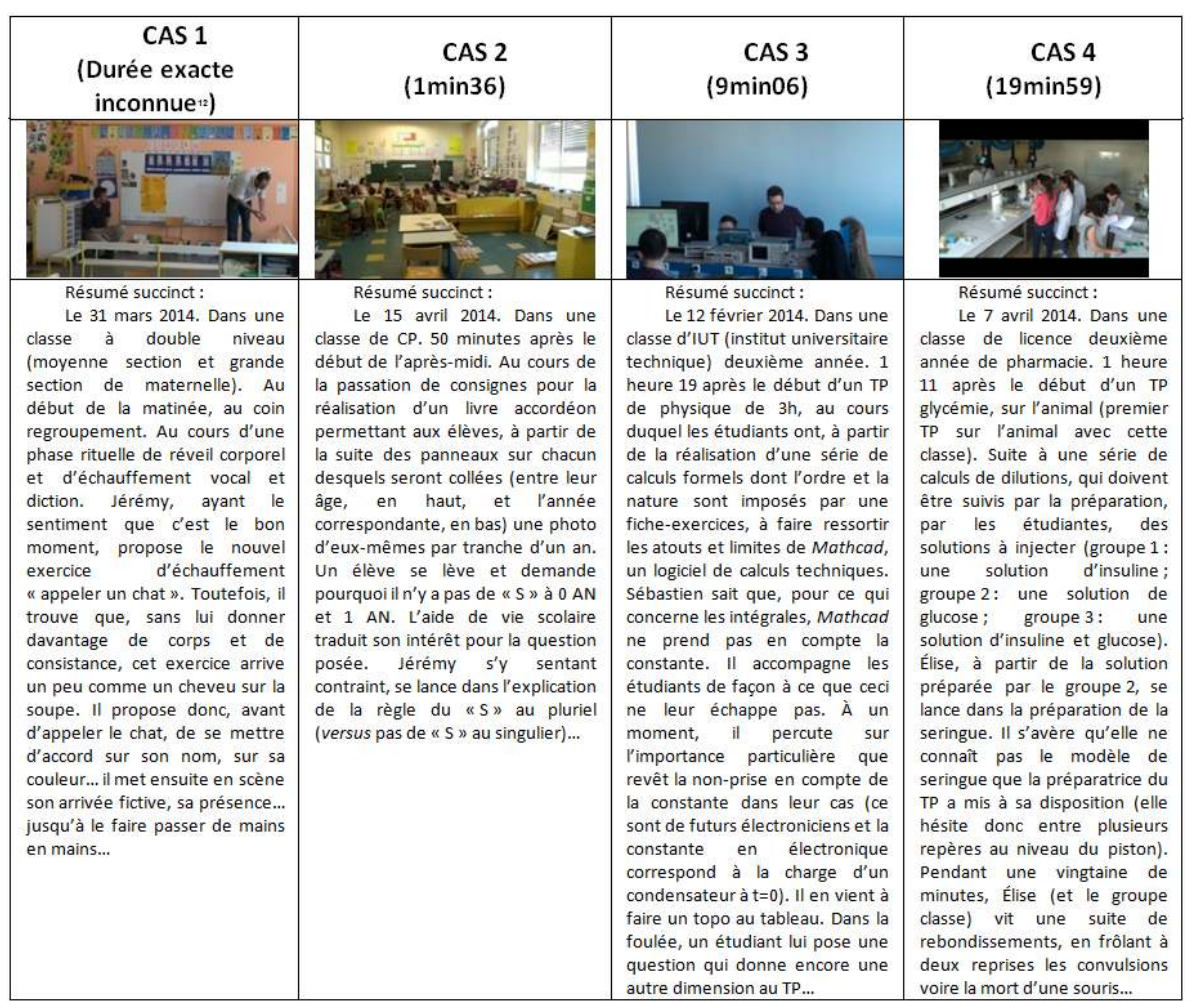

Note $^{12}$ : Du fait que nous avons ici opéré par remise en situation dynamique, sans traces vidéographiques et sans avoir observé l'interaction en question.

Les résultats associés à ces études de cas mettent notamment en exergue 4 catégories spécifiques: la nouveauté, la surprise, l'incertitude et l'apprentissage-développement, associé à l'activité-improvisation. Soulignons le fait suivant : les différentes dimensions dont il est question ci-après doivent être considérées comme tissées ensemble et non pas comme des dimensions isolées qui à elles seules feraient l'estimation improvisation de l'activité. La nouveauté constitue la clé du complexe. 


\subsubsection{Nouveauté}

La dimension nouveauté émerge à l'analyse :

- De signes dont les unités élémentaires d'actions ont de l'ordre du jamais vécu, du jamais fait. Par exemple, Jérémy sur fond du début d'une forme de liste au tableau, fait le lien avec les élections municipales qui ont eu lieu le week-end précédent, et a l'idée de faire un vote (chose qu'en classe, avec des élèves de moyenne section, il n'a jamais faite);

- Et/ou de signes pour lesquels il existe un delta (au moins partiel) entre la structure d'anticipation + le référentiel, et le representamen et l'unité. Par exemple dans le CAS 2 du tableau 1 (voir le tableau 2, ci-après). Jérémy est préoccupé par la fonctionnalité de la règle telle qu'énoncée (avec ces élèves de CP et pour le plus grand nombre d'élèves), ainsi que par le fait d'achever l'illustration de la règle orthographique; alors que la structure d'anticipation corrélative de ses préoccupations se porte sur cette fonctionnalité et sur le fait de l'achèvement de l'illustration, l'absence et la présence $d u$ « $s$ » aux mots POMME(S) fait signe sous un jour nouveau - dans le sens où ce signe est en décalage relatif avec la structure d'anticipation $\mathrm{A}$ et en décalage marqué avec le référentiel. Ce signe est parallèlement constitué de développement de savoirs;

- Et/ou de signes dont l'interprétant correspond à une découverte de savoir ou à une émergence de type. Par exemple, l'interprétant du signe 85 du CAS 3 du tableau 1 est le suivant: Découverte de savoir: [Le mode de calcul de Mathcad]: amène à perdre un polynôme. 
Tableau 2: CAS2, détail du signe 62.

Table 2: CAS2, detail of sign 62

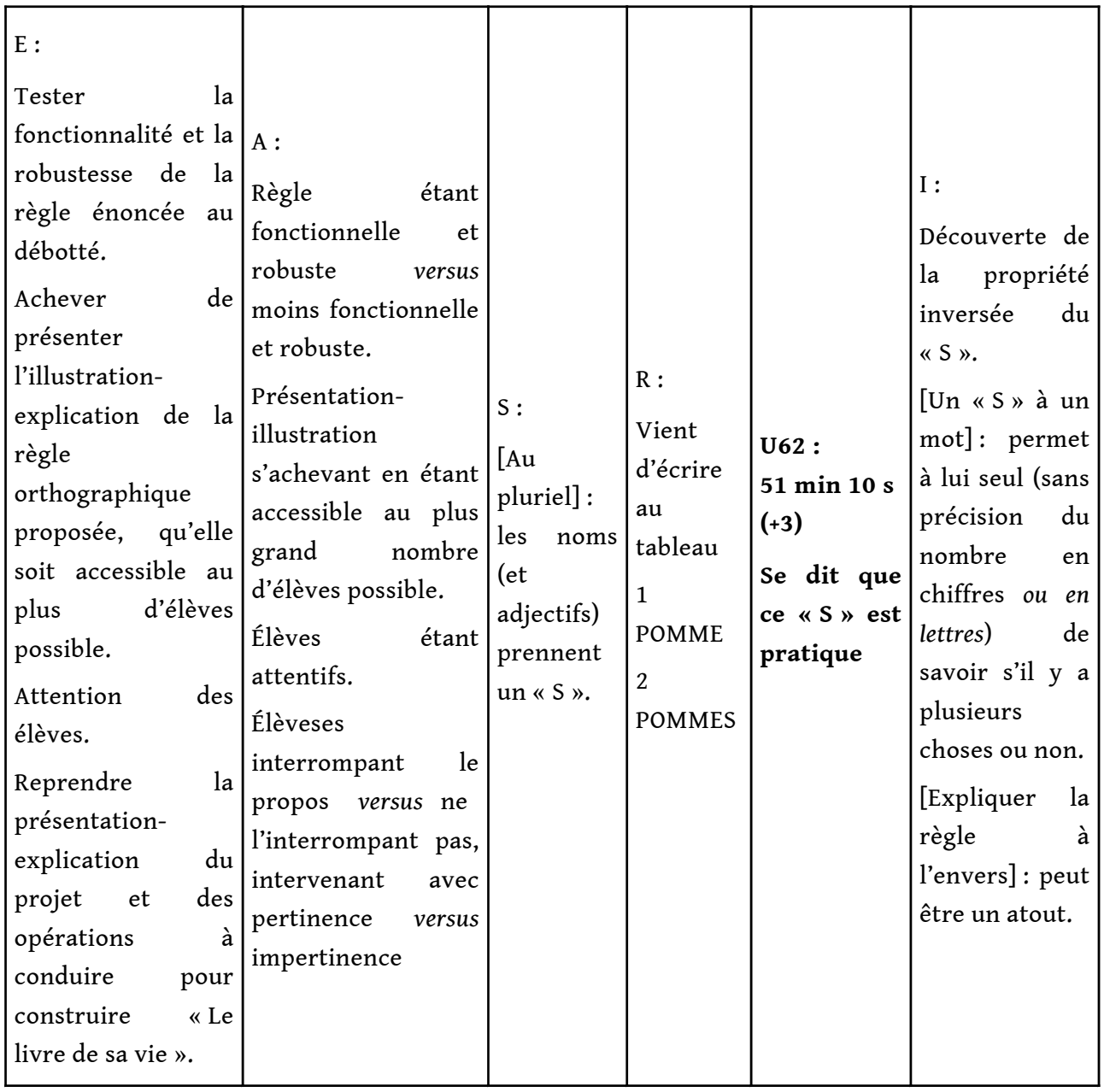

\subsubsection{Surprise}

La dimension surprise émerge à l'analyse du rapport pour certains signes entre : a) le repérage d'un delta marqué entre la structure d'anticipation et le representamen et/ou l'unité de cours d'action (il apparait en effet que la surprise trouve sa source dans l'impossibilité pour l'acteur de voir se déployer une structure d'anticipation comprenant des anticipations de representamens ou d'unités proches ou correspondant aux occurrences effectives), et b) la communication au cours de l'autoconfrontation, d'un sentiment de surprise par l'acteur (qu'elle soit prosodique, mimo-gestuelle ou verbale) reporté dans les unités de cours d'action. Par exemple dans le CAS 1 du tableau 1 (voir le Tableau 3, ci-après) : 
Tableau 3 : CAS1, détail du signe 32.

Table 3: CAS1, detail of sign 32

\begin{tabular}{|c|c|c|c|c|c|}
\hline $\begin{array}{l}\text { E : } \\
\text { Maintien des } \\
\text { élèves dans la } \\
\text { scénarisation } \\
\text { de l'exercice- } \\
\text { jeu. } \\
\text { Fonder le } \\
\text { groupe classe. }\end{array}$ & $\begin{array}{l}\text { A : } \\
\text { Élèves restant } \\
\text { dans versus ne } \\
\text { restant pas } \\
\text { dans la } \\
\text { scénarisation. } \\
\text { Faire semblant } \\
\text { débouchant } \\
\text { sur des } \\
\text { occurrences } \\
\text { d'une certaine } \\
\text { nature. }\end{array}$ & $\begin{array}{l}\text { S : } \\
\text { [Élèves vivant } \\
\text { pleinement la } \\
\text { scène] : } \\
\text { implique de } \\
\text { soi-même } \\
\text { avoir } \\
\text { l'impression } \\
\text { d'y être, et } \\
\text { d'être précis } \\
\text { dans sa } \\
\text { gestuelle. } \\
\text { [Un bon } \\
\text { moment]: est } \\
\text { encore plus } \\
\text { fort s'il est } \\
\text { pleinement } \\
\text { partagé. }\end{array}$ & $\begin{array}{l}\text { R: } \\
\text { Précision des } \\
\text { sensations, } \\
\text { notamment celles } \\
\text { liées au toucher, à } \\
\text { la forme du } \\
\text { chat imaginaire. }\end{array}$ & $\begin{array}{l}\text { U32: } \\
\text { Surpris } \\
\text { par la } \\
\text { teneur } \\
\text { de son } \\
\text { propre } \\
\text { vécu. } \\
\text { « Vous } \\
\text { voulez le } \\
\text { caresser } \\
\text { aussi » }\end{array}$ & $\begin{array}{l}\text { I : } \\
\text { Émergence du } \\
\text { type } \\
\text { [Lors de cette } \\
\text { scène fictive, } \\
\text { les sensations, } \\
\text { notamment } \\
\text { en lien avec le } \\
\text { toucher]: } \\
\text { peuvent être } \\
\text { très précises. }\end{array}$ \\
\hline
\end{tabular}

La nouveauté et la surprise concernent tout autant ce que, dans le cours du couplage à son environnement, l'acteur rencontre, que ce qui lui vient ou ce qu'il vit, que ce qui se passe (qu'il soit plus ou moins au cœur de ce qui se déploie).

\subsubsection{Incertitude}

La dimension incertitude émerge à l'analyse : a) de signes dont les unités de cours d'action de l'acteur traduisent clairement l'incertitude ou le doute: par exemple, CAS $1 \mathrm{du}$ tableau 1, Jérémy se dit qu'il va faire passer le chat imaginaire aux élèves tout en se demandant s'ils vont tenir jusqu'au bout lorsque les premiers l'auront eu, b) de signes dont l'interprétant correspond à une mise en question du savoir déjà construit - par exemple dans le CAS 3 du tableau 1 (voir Tableau 4, ci-après). 
Tableau 4 : CAS3, détail du signe 75.

Table 4: CAS3, detail of sign 75

\begin{tabular}{|c|c|c|c|c|c|}
\hline $\begin{array}{l}\text { E : } \\
\text { Comprendre } \\
\text { la question } \\
\text { de G7E2 } 2^{13} \text {. } \\
\text { "Se } \\
\text { brancher } \\
\text { au " calcul } \\
\text { interrogeant } \\
\text { G7E2. }\end{array}$ & $\begin{array}{l}\text { A : } \\
\text { G7E2 posant } \\
\text { une question } \\
\text { en relation } \\
\text { avec ce qui } \\
\text { vient d'être } \\
\text { dit, } \\
\text { demandant } \\
\text { une } \\
\text { vérification } \\
\text { de calcul. }\end{array}$ & $\begin{array}{l}\text { S: } \\
\text { [Comprendre une } \\
\text { question } \\
\text { mathématiques]: } \\
\text { suppose de se } \\
\text { repérer dans les } \\
\text { calculs. }\end{array}$ & \begin{tabular}{|l} 
R: \\
G7E2: Est- \\
ce que je \\
prends en \\
compte la \\
constante \\
quand je \\
vais \\
intégrer la \\
double \\
intégrale de \\
sinus oméga \\
$t ?$
\end{tabular} & $\begin{array}{l}\text { U75 : } \\
1 \text { h } 30 \text { min } 28 \mathrm{~s} \\
(+4) \\
\text { Surpris } \\
\text { Regarde-lit le } \\
\text { calcul pointé } \\
\text { par G7E2. } \\
\text { Réfléchit }\end{array}$ & $\begin{array}{l}\text { I : } \\
\text { [La question } \\
\text { de l'élève et } \\
\text { le calcul à } \\
\text { réaliser]: } \\
\text { interrogent }\end{array}$ \\
\hline
\end{tabular}

Note $^{13}$ : Comprendre Groupe 7 Elève 2.

47 Selon les cas, la dimension incertitude est à corréler à une possible mise en danger plus moins forte pour l'acteur : a) danger de perte de contrôle du groupe classe (CAS 1 et 2); b) danger d'une déconsidération par l'aide de vie scolaire (et ses collègues) (CAS 2); c) danger d'un traumatisme des étudiants (CAS 4, en cas de mort de la souris); d) danger d'une déconsidération de l'enseignant par les élèves ou étudiants (CAS 2, 3 et 4).

\subsubsection{Apprentissage-développement}

Ce sont les interprétants qui, lors de l'analyse, nous permettent de faire émerger les informations sur l'apprentissage-développement dans la dynamique située du couplage acteur-situation.

Dans tous les cas, les interprétants Découverte de type sont systématiques. Par exemple :

- CAS 1 du tableau 1, Jérémy découvre qu'au coin regroupement ces élèves de maternelle sont capables de rester calmes et attentifs sur un temps long et de se respecter lors du passage de mains en mains d'un chat imaginaire ;

- CAS 2, Jérémy découvre que pour une première approche de la règle orthographique du « $\mathrm{S} » /$ Pas de « $\mathrm{S}$ », le rapport d'opposition «Plusieurs/Pas plusieurs» est préférable à singulier/pluriel;

- CAS 3 Sébastien découvre que le mode de calcul du logiciel Mathcad amène à perdre un polynôme ;

- CAS 4, Élise découvre comment lire le volume sur des seringues inconnues, ou que suite au calcul de dilution concernant les solutions à injecter, les étudiantes sont capables d'oublier d'effectivement faire les dilutions malgré la fiche guide du TP.

Si ces découvertes peuvent apparaître jaillissantes à un certain moment, comme une bulle qui claque à la surface, elles se déploient aussi progressivement dans une série d'autres découvertes, de doutes, d'inférences, de renforcement de validité (pour plus de détail, voir l'annexe 2). 
51 La présentation de la dimension apprentissage-développement vient conclure l'exposé d'un complexe crucial qui, selon les enseignants entrant dans le métier, caractérise l'activité-improvisation. Outre ces 4 dimensions cruciales, il nous paraît important de relever que le régime de l'activité-improvisation apparaît spécifique.

\subsubsection{Une activité à régime « dilaté »}

52 Nous revenons autrement ici sur le CAS 2 du tableau 1, 37 secondes après le début de l'épisode estimé improvisé par Jérémy. Sous le regard de l'ensemble des élèves présents et de l'AVS, il se sent contraint de répondre à une question d'un élève, dont il estime qu'elle arrive « comme un cheveu sur la soupe » et qu'elle "n'a rien à voir avec ce qu'ils font ». Alors qu'il est en train de passer une consigne compliquée destinée à la réalisation du «Livre de leur vie» (pour lequel ils vont devoir coller sur une frise, des photos d'euxmêmes à différents âges), Maëvan se lève et lui demande pourquoi il n'y a pas de " $S$ » à 0 AN et 1 AN sur le canevas au tableau. Jérémy, qui a entendu la réaction de l'AVS (voir le tableau 1, partie cadre méthodologique), se lance au débotté dans une explication de la présence $\mathrm{du}$ « $\mathrm{S} »$ au pluriel.

53 La question de Maëvan et la réaction de l'AVS ont surpris Jérémy, "pris au dépourvu ». Son niveau attentionnel est monté d'un cran, dans le cours d'une interaction complexe et d'une activité qu'il vit comme condensée. En comparaison à ce qui le précède, il vit plus intensément ce moment d'interaction, avec les autres (avec l'AVS, qu'il prend en compte et à laquelle il s'adresse indirectement ; avec les élèves, auxquels il s'adresse directement) et avec lui-même. Il prend le temps de choisir ses mots de manière à ce que son explication soit à la hauteur (entre autres de l'attente supposée de l'AVS). Jugeant que le mot AN est trop abstrait pour que des élèves de CP fassent le lien nombre/chose, qui conditionne la règle $d u$ « $S$ » au pluriel dans la langue française, il recherche un autre mot. Lui viennent d'abord l'image et le mot « cheval», dont il se dit que cela « ne marche pas du tout ", puis le mot "cerf-volant». Un peu paniqué, un peu énervé contre luimême, vivant un tourbillon intérieur, dans une série de gestes invisibles, il tente de se relâcher, «d'enlever, virer, vider » les mots qui ne conviennent pas, « de se débarrasser du poids de ces mots » pour laisser la place à un mot qui va, il se dit « pense simple »...

Cet exemple illustre le fait que, dans le cas des épisodes d'improvisation étudiés, l'activité change de « régime " (Billeter, 2010). Il apparaît que ce changement de régime doit être mis en relation avec une variation attentionnelle, interactionnelle, émotionnelle, ou « réflexive » (pour reprendre le terme de Schön, 1994) et qu'il est le corrélat du complexe impulsé par la nouveauté de ce que rencontre l'acteur et/ou de ce qui lui vient et/ou de ce qui arrive.

\subsection{Renforcement et/ou enrichissement des résultats}

Tout au long du travail ethnographique et dans le jeu de proximité/distance dans lequel il engage le chercheur, nous avons dû faire avec le fait que, par moments, nous mobilisions par-devers nous certaines de nos propres catégories expérientielles et culturelles concernant l'improvisation ${ }^{14}$. À première vue, ce que nous montraient ou nous disaient les acteurs pouvait nous paraître illogique. Nous pointions alors des étrangetés et des incohérences pour nous, qui, paradoxalement, ont contribué à la compréhension de l'activité-improvisation. C'est en effet en acceptant aussi une forme de tension entre le 
pour nous et le pour l'autre, que nos résultats ont pris du relief. Étant entendu que c'est le point de vue et la réalité des autres, l'analyse de leur activité, qui a permis de construire les catégories citées ci-avant.

\subsubsection{Banalité versus nouveauté} pré-vu (vu avant) ce qu'ils jouaient dans l'interaction. Dont nous jugions du coup, qu'elle était paradoxalement estimée improvisée. Pour reprendre certaines illustrations exposées plus haut : Flora : «C'est un truc que j'avais vu dans une vidéo à l'IUFM »; Cindy : «Je l'avais vu faire par ma tutrice".

61 Dans ces cas où les acteurs ont déjà vu ce qui leur vient, ce qui est nouveau correspond au fait que c'est la première fois qu'ils/elles actualisent ce qui a été vu avant et ailleurs. Et ce qui est surprenant pour les acteurs est lié à la spontanéité de ce qui leur vient, dans un espace-temps où ils/elles ne l'attendent pas.

\subsubsection{Incertitude versus confiance}

Nous avons été interpellés par un double jeu entre incertitude et confiance. Nous l'avons évoqué, les néo-enseignants ont besoin de se sentir en confiance pour aller vivre un cours. Pour cela ils ont régulièrement besoin de se sentir à l'aise avec le contenu enseigné et/ou l'espace d'enseignement (classe, amphi), le matériel, leurs assistants (ATSEM, AVS, binôme de TP, etc.), etc. Cette aisance minimale permet d'oser affronter une situation à forte probabilité de contingence, mais aussi une situation dont ils savent par expérience qu'elle a de grandes chances d'être déstabilisante; l'idée étant alors de limiter, par anticipation, le degré de déstabilisation. Finalement c'est, pourrait-on dire, autant 
l'incertitude que la certitude qui, ici, peut être la source d'une inquiétude et d'une recherche de confiance suffisante. l'incertitude ne s'y oppose pas à la certitude (ou à la confiance) ou plutôt, l'incertitude ne débouche pas de manière mécanique et systématique sur une absence de certitude, de conviction (ou de confiance). Ils disent à la fois ne pas (du tout) savoir où va l'interaction, ce qu'elle va donner, ni si ce qui leur est venu est pertinent, mais ils se disent aussi confiants et, dans certains cas, convaincus de bien sentir ce qui leur est venu ou ce qui se passe. D'autre part, il faut noter que même lorsque les néo-enseignants ne font référence qu'à l'incertitude, c'est une incertitude qui ne semble pas les gêner ou bloquer le processus lui-même. L'analyse des illustrations nous conduit à proposer 4 compréhensions du phénomène :

- La spontanéité ou l'effet de jaillissement de ce qui vient aux acteurs ne laisse plus, au moment, de place au doute, et est accompagné d'un éclair de conviction ;

- Pour faire, il faut qu'ils se convainquent a minima. Puis l'action, et un changement d'attention à la situation, prennent le pas sur le doute. Agnès : "Je sais pas où est-ce qu'on va aller. Sur le moment on sait pas où on va et puis après c'est lancé! » Damien : "T'as tous tes sens en éveil »; Agnès : "J'ai l'impression de faire plus attention»; Stéphanie : "Je suis attentive d'une autre manière en fait»; Jérémy: "T'es plus à l'écoute en fait»; Muriel : "On a une certaine adrénaline (...) On est boosté (...) plus présent »;

- En cours d'interaction, ils vivent des indicateurs positifs. Stéphanie : «Tu sens une atmosphère de classe très différente en fait », etc. ;

- À l'issue d'une activité-improvisation, qui accompagne une interaction qui n'était pas gagnée d'avance, l'acteur éprouve le sentiment de sortir grandi, il se sent plus confiant parce qu'il se sait effectivement capable. Agnès : "En plus c'est peut-être étonnant venant de notre part, on va dire les novices (...) où on nous dit voilà faut tout préparer et ci et mi, et alors que justement quand on fait une expérience justement un peu de se laisser aller, ben on se rend compte que c'était pas si désagréable (...) ça ramène de la confiance».

\subsubsection{Fuir versus rechercher l'activité-improvisation} l'improvisation. La situation d'interaction en classe est perçue comme une situation à danger potentiel. C'est alors une improvisation pouvant déboucher sur une trop grande
déstabilisation, voire une « galère ", qui est attendue. Tout est mis en œuvre pour limiter
autant que faire se peut son intrusion. L'idée est alors d'avoir la main sur un maximum de
paramètres. Ils ont souvent la tentation de verrouiller la préparation en laissant le moins
de place possible à l'impondérable (par exemple, en CM, ils prévoient du volume à
débiter, certains font tout pour éviter les temps morts, synonymes de questions
potentiellement déstabilisantes... voire ils s'arrangent pour prendre du retard dans le
démarrage du cours, retard qui justifiera un débit élevé...). Quelles que soient les formes danger potentiel. C'est alors une improvisation pouvant déboucher sur une trop grande
déstabilisation, voire une « galère », qui est attendue. Tout est mis en cuvre pour limiter
autant que faire se peut son intrusion. L'idée est alors d'avoir la main sur un maximum de
paramètres. Ils ont souvent la tentation de verrouiller la préparation en laissant le moins
de place possible à l'impondérable (par exemple, en CM, ils prévoient du volume à
débiter, certains font tout pour éviter les temps morts, synonymes de questions
potentiellement déstabilisantes... voire ils s'arrangent pour prendre du retard dans le
démarrage du cours, retard qui justifiera un débit élevé...). Quelles que soient les formes danger potentiel. C'est alors une improvisation pouvant déboucher sur une trop grande
déstabilisation, voire une " galère », qui est attendue. Tout est mis en œuvre pour limiter
autant que faire se peut son intrusion. L'idée est alors d'avoir la main sur un maximum de
paramètres. Ils ont souvent la tentation de verrouiller la préparation en laissant le moins
de place possible à l'impondérable (par exemple, en CM, ils prévoient du volume à
débiter, certains font tout pour éviter les temps morts, synonymes de questions
potentiellement déstabilisantes... voire ils s'arrangent pour prendre du retard dans le
démarrage du cours, retard qui justifiera un débit élevé...). Quelles que soient les formes danger potentiel. C'est alors une improvisation pouvant déboucher sur une trop grande
déstabilisation, voire une " galère », qui est attendue. Tout est mis en œuvre pour limiter
autant que faire se peut son intrusion. L'idée est alors d'avoir la main sur un maximum de
paramètres. Ils ont souvent la tentation de verrouiller la préparation en laissant le moins
de place possible à l'impondérable (par exemple, en CM, ils prévoient du volume à
débiter, certains font tout pour éviter les temps morts, synonymes de questions
potentiellement déstabilisantes... voire ils s'arrangent pour prendre du retard dans le
démarrage du cours, retard qui justifiera un débit élevé...). Quelles que soient les formes danger potentiel. C'est alors une improvisation pouvant déboucher sur une trop grande
déstabilisation, voire une « galère », qui est attendue. Tout est mis en œuvre pour limiter
autant que faire se peut son intrusion. L'idée est alors d'avoir la main sur un maximum de
paramètres. Ils ont souvent la tentation de verrouiller la préparation en laissant le moins
de place possible à l'impondérable (par exemple, en CM, ils prévoient du volume à
débiter, certains font tout pour éviter les temps morts, synonymes de questions
potentiellement déstabilisantes... voire ils s'arrangent pour prendre du retard dans le
démarrage du cours, retard qui justifiera un débit élevé...). Quelles que soient les formes danger potentiel. C'est alors une improvisation pouvant déboucher sur une trop grande
déstabilisation, voire une « galère », qui est attendue. Tout est mis en cuvre pour limiter
autant que faire se peut son intrusion. L'idée est alors d'avoir la main sur un maximum de
paramètres. Ils ont souvent la tentation de verrouiller la préparation en laissant le moins
de place possible à l'impondérable (par exemple, en CM, ils prévoient du volume à
débiter, certains font tout pour éviter les temps morts, synonymes de questions
potentiellement déstabilisantes... voire ils s'arrangent pour prendre du retard dans le
démarrage du cours, retard qui justifiera un débit élevé...). Quelles que soient les formes danger potentiel. C'est alors une improvisation pouvant déboucher sur une trop grande
déstabilisation, voire une « galère », qui est attendue. Tout est mis en cuvre pour limiter
autant que faire se peut son intrusion. L'idée est alors d'avoir la main sur un maximum de
paramètres. Ils ont souvent la tentation de verrouiller la préparation en laissant le moins
de place possible à l'impondérable (par exemple, en CM, ils prévoient du volume à
débiter, certains font tout pour éviter les temps morts, synonymes de questions
potentiellement déstabilisantes... voire ils s'arrangent pour prendre du retard dans le
démarrage du cours, retard qui justifiera un débit élevé...). Quelles que soient les formes danger potentiel. C'est alors une improvisation pouvant déboucher sur une trop grande
déstabilisation, voire une « galère ", qui est attendue. Tout est mis en œuvre pour limiter
autant que faire se peut son intrusion. L'idée est alors d'avoir la main sur un maximum de
paramètres. Ils ont souvent la tentation de verrouiller la préparation en laissant le moins
de place possible à l'impondérable (par exemple, en CM, ils prévoient du volume à
débiter, certains font tout pour éviter les temps morts, synonymes de questions
potentiellement déstabilisantes... voire ils s'arrangent pour prendre du retard dans le
démarrage du cours, retard qui justifiera un débit élevé...). Quelles que soient les formes 
qu'elles revêtent (plus ou moins académiques, laissant ou non des traces écrites), les préparations sont longues, fouillées. Réalisées à la maison ou sur le lieu de travail, elles occupent les moindres zones de temps libre, parfois jusque tard dans la nuit. Il faut se sentir en confiance. Malgré tout, l'improvisation s'invite régulièrement, au moment de jouer effectivement la « partition », avec le « public ».

"Ras le bol des aléas!", les néo-enseignants ont aussi besoin de routines. Emeline: "ça change tout le temps, vivement que j'aie des choses dont je sois sûre, et que je vais pouvoir faire un peu en routine ». En même temps, on remarque un double discours: au début on est très loin de vouloir improviser, et dès que possible (c'est-à-dire dès que la confiance en soi et en la situation est suffisante, ce qui est différent de maximale, et dès que la situation s'y prête) on recherche l'activité-improvisation (y compris les enseignants stagiaires)... en s'autorisant des digressions partielles, en allant parfois en cours sur la base d'une préparation plus légère, voire en y allant «free-lance » (ce qui ne signifie pas une absence de préparation, mais une absence de préparation écrite). Au moins trois paramètres semblent participer à la recherche de l'activité-improvisation :

- Ils appréhendent la sclérose, et ce qu'ils anticipent de ce qui l'accompagne: baisse d'opérationnalité, perte de plaisir, etc. Certains d'entres eux expriment clairement qu'ils fuient les effigies qu'ils se sont construites au gré de leurs expériences d'élèves, d'étudiants ou de néo-professionnels, d'enseignants englués dans leurs routines qui ne trouvent plus de sens à ce qu'ils font, et dont le don est d'endormir les élèves ou d'oublier leur présence jusqu'à leur manquer de respect. Curieusement, cette sclérose, ils la découvrent euxmêmes ;

- Contre toute attente, en effet, ils sont aussi régulièrement pris dans des routines, des habitudes qui, si elles sont activement recherchées, en viennent aussi à les ennuyer (c'est le cas pour les enseignements et les situations d'enseignement qui très vite se répètent et perdent de leur sens, si tant est qu'à un moment ils en aient eu réellement un : e.g. les rituels du coin regroupement en maternelle, une forme de cours redondante dans le second degré, tel ou tel TP dans l'enseignement supérieur - ils disent ressentir que cet ennui déteint sur le groupe classe, et qu'inversement les temps d'activité qu'ils appellent improvisation sont plus vivants, vivifiants). D'autre part, il faut noter que ce qui est habituel, routinier peut être une forme d'interaction qui ne correspond pas à leurs attentes ou à leurs visées, qui ne leur ressemble pas et peut les faire souffrir (par exemple : une certaine façon de marquer son autorité). (Re)créer les conditions de la surprise, de la nouveauté, devient alors l'issue à l'enfermement dans un travail qui peut blaser, ou tourner en boucle dans une dynamique insatisfaisante, voire délétère. Emeline : « Ça fait sortir du carcan habituel du TD »;

- Ils ont besoin de tenter par eux-mêmes, d'aborder des sujets et de mettre en œuvre des formats pédagogiques qui leur tiennent à cœur ou qu'ils veulent tester (parfois depuis longtemps). Audacieux, les néo-enseignants, comme des héros de contes, dérogent aussi à la règle, font fi des «menaces de loup ", pour tenter « le déconseillé » ou l'interdit, ce que l'on n'est pas censé faire. Soit parce que cela «les titille», soit aussi, par la force des choses (Jérémy : qui dans une classe de maternelle inattentive à sa lecture, prend le livre et le jette sur le côté pour, au lieu de lire l'histoire, la raconter en la théâtralisant).

L'activité-improvisation, réputée énergivore et risquée, est aussi recherchée pour se ressourcer, s'économiser, se stimuler, se construire.

En synthèse, les principaux résultats tiennent en peu de mots. Au cours d'une interaction à la fois marquée par la régularité, par l'inhabituel et par le hasard, liée à des moments « uniques », l'activité-improvisation apparait comme une activité : a) dont le gradient de 
nouveauté, de surprise et d'incertitude est élevé - corrélatif de ce que rencontrent les néo-enseignants et/ou de ce qui leur vient et/ou de ce qui se passe; b) dont le régime, interactionnel, attentionnel et émotionnel est spécifique ; et c) au cours desquels, comme dans une sorte de «saut brusque ( (Simondon, 2013), sont découverts des savoirs, sont générées (ou ré-générées, soit générées autrement, en s'actualisant) des « dispositions à agir » (Muller, 2014), dont la preuve d'une certaine efficacité est faite ici et maintenant, " en direct live ». Parmi ces dispositions, on peut considérer qu'ils entretiennent et/ou développent deux formes de "méta-dispositions» consistant en: une disposition à l'attention et une disposition à la transformation. Ces moments participent donc de façon majeure à leur apprentissage-développement.

\section{Discussion}

70 Finalement, en s'appuyant sur l'ensemble de ce qui précède on pourrait considérer que, pour les néo-enseignants, il n'y a pas de construction de soi ni de développement professionnel sans « improvisation », en classe.

71 Durand et Perrin (2014, p. 29) conçoivent la notion d'individuation, empruntée à Simondon, comme « le processus par lequel se construisent les individus au cours de leur activité et de l'histoire du couplage avec leur environnement ». Pour Simondon (2013): (a) l'individu (conçu comme « résultant de ", donc second,) est l'expression momentanée d'un processus d'individuation (conçu comme premier), (b) l'individu est une phase de l'être, lui-même considéré comme l'équivalent d'un germe, d'un potentiel, dont le devenir est l'une des dimensions, (c) l'individuation est une médiation qui permet le passage de l'être à l'individu, jamais achevée, car «elle n'épuise pas d'emblée les ressources potentielles de l'être» (Ibid., p. 315). Autrement dit, l'individu est le résultat d'un processus continu, sans cesse inachevé, du fait du couplage à son environnement, ad vitam et ad mortem.

Un système stable étant un système ayant atteint le plus haut degré d'homogénéité possible, il ne peut en aucun cas expliquer l'action, «car il est le système dans lequel aucune transformation n'est possible puisque tous les potentiels sont épuisés: il est système mort » (Ibid., p. 212). Considérer l'activité-improvisation comme corrélative d'un espace-temps spécifique d'individuation, c'est, fondamentalement, l'assimiler à une activité "poiétique ${ }^{15}$ ", c'est-à-dire à une activité de création, d'invention et de (re)génération non seulement de pensées, d'actions ou de choses particulières, mais de soi. Sans laquelle, nous semble-t-il, l'activité court le risque de la sclérose, voire de la nécrose, et l'acteur celui de devenir à terme, un automate voire un spectre.

Comment, dès lors penser à nouveau frais les questions de formation au métier d'enseignant ? Cette discussion tentera de fournir quelques pistes.

Nos résultats nous invitent, à l'instar de Ria $(2009,2012)$, à réaffirmer la capabilité des néo-enseignants, et à nous inscrire fermement dans le principe d'égalité des intelligences ${ }^{16}$, développé par Rancière (1987), et posé par lui comme condition première de l'égalité effective, et source de développement des hommes et des femmes de structures et d'une société effectivement égalitaire. Dans l'ensemble, nous défendrons principalement l'idée que la formation à l'activité-improvisation est d'un certain point de vue impossible, mais que l'accompagnement au développement de dispositions à agir, pouvant être utiles à cette dernière, est à la fois possible et souhaitable. Ce faisant, nous escomptons éviter le 
double écueil souligné par De Raymond (1980), concernant l'apprentissage de l'improvisation. Il écrit :

«cet apprentissage porte le risque d'une détention: qui prétend que l'improvisation s'apprend, sinon ceux qui veulent maintenir la prégnance d'un modèle d'acculturation et donc lui ôter son caractère explosif ? Ou inversement, qui dissuadera de l'apprentissage de l'improvisation, sinon ceux qui veulent la réserver à une catégorie d'individus? » (Ibid., p. 65).

\subsection{Pourquoi l'activité-improvisation peut-elle difficilement faire l'objet d'une formation ou d'une professionnalisation}

Selon nous, il résulte de ses caractéristiques, une difficulté intrinsèque, plus spécifique à l'activité-improvisation, à se voir convertie en l'objet d'une formation considérée selon le schéma hylémorphique et s'apparentant à une professionnalisation (à savoir, comme relevant de l'intention orientée d'une institution de mettre en mouvement ses personnels, Wittorski \& Briquet-Duhazé, 2009). En effet, l'activité-improvisation, sans doute davantage que toute autre, résiste à cette idée de donner au formé une (bonne) forme, de tendre vers un état, connu d'un tiers déjà compétent, déjà connaissant, et souhaité par lui/elle et/ou par l'institution dans laquelle il œuvre. Par ailleurs, on peut logiquement s'entendre sur le fait que la nouveauté est à chaque fois unique, or, "peuton apprendre ce qui, par définition, ne se répète pas, qui à chaque fois diffère, n'arrive qu'une fois tel qu'il arrive ?» interroge De Raymond (1980, p. 62).

On peut en revanche considérer que la dynamique propre à ce type d'interaction en classe, les actualisations qui s'opèrent en son cours ainsi que l'expérience qui l'accompagne, sont sources de développement professionnel (donc d'une « dynamique de développement que met en œuvre un individu au fil des situations de travail et de formation qu'il rencontre ", Wittorski \& Briquet-Duhazé, 2009, p. 45). Et qui constituent la formation elle-même, entendue cette fois comme «un processus (...), la synthèse dynamique et complexe d'activité ou d'interactivité » (Durand, 2008, p. 109).

On peut ainsi soutenir que l'activité-improvisation, en tant qu'activité d'abord développementale, résistante et libre, apparaît comme un possible antidote à la déprofessionnalisation, et autres dégradations (individuelles ou sociales). Nos résultats nous la donnent entre autres à voir, en arrière de toute forme d'orthodoxie, comme une " activité silencieuse, transgressive, ironique ou poétique [d'acteurs ${ }^{17}$ ] qui conserve leur quant-à-soi dans le privé et à l'insu des "maîtres" " (De Certeau, 1990, p. 249). Elle correspond enfin à l'un de ces espaces et de ces temps dans lesquels se jouent le brassage et le métissage de la culture des acteurs en présence, «au risque de l'autre » (MabilonBonfils \& Zoïa, 2014) - œuvre démocratique.

Précisons encore que si «l'improvisation déjoue les machinations qui cherchent à secondariser l'action en la soumettant à des conditions de temps qui la reculent à l'infini, aux méthodologies qui la brident ou la désamorcent, ou aux orthodoxies qui neutralisent son originalité. » (De Raymond, 1980, p. 123), elle ne présente pas moins des régularités, qui font ses caractéristiques. Nous retiendrons que l'activité-improvisation est impossible à téléguider, voire à assagir, mais qu'il est sans doute possible et souhaitable, de considérer son existence selon les néo-enseignants, d'accompagner son émergence et l'entretien de son mouvement. D'autant qu'on peut être malheureux si l'on joue toujours la musique de quelqu'un d'autre (Paco Peña, in Bailey, 2011), ou qu'on peut se demander 
quelle raison il y a à exister, si l'on ne participe pas à la créativité de son époque (Anthony Pay, in Bailey, 2011).

«On ne s'improvise pas improvisateur ». Il y a au moins trois façons d'entendre cette maxime : la première consiste à défendre qu'il faille d'abord étudier et connaître, en théorie, et incrémenter un répertoire d'unités discrètes d'actions, pour ensuite s'essayer à l'improvisation. La deuxième, que le savoir-faire de l'improvisateur se développe par la pratique de l'improvisation elle-même, que l'expérience de l'improvisation fait à elle seule tout le savoir-faire. La troisième, que le savoir-faire de l'improvisateur se construit dans l'alternance entre l'improvisation elle-même (en tant qu'activité ou forme culturelles expérimentées dès le départ) et la pleine participation à une culture générale annexe (donc des cultures), qui l'irriguent, l'inspirent, la débordent. Nos résultats concernant l'activité-improvisation nous invitent à nous ranger dans la troisième catégorie, donc à défendre que la formation à l'activité-improvisation soit impossible, mais que l'accompagnement de son émergence, et de la construction de dispositions à agir participant du déploiement de son plein potentiel, soit à la fois possible et souhaitable.

\subsection{Offrir d'emblée la possibilité de l'émergence de l'activité-improvisation}

80 Précisons que cette question de justifier en quoi il est souhaitable d'offrir d'emblée aux futurs et néo-enseignants, la possibilité de l'émergence d'une activité-improvisation en interaction en classe, nous interpelle d'autant plus du fait de la fréquence avec laquelle les néo-enseignants du premier et du second degré soulignent l'écart et le déséquilibre entre formation «théorique » et formation « pratique» (e.g. Geay, 2010 ou Rayou \& Van Zanten, 2004). Ce qui peut laisser à penser que, malgré les efforts des institutions spécialisées dans la formation, pour que soit mise en place une alternance vertueuse entre théorie et pratique, quelque chose continue de "pêcher ».

81 Nous pensons que considérer l'improvisation comme réservée à demain, une fois incrémentée une somme de savoirs théoriques décontextualisés, c'est sous-estimer a priori l'expérience fondatrice de la corrélation, dans le même temps, de la réflexion et de l'action. Il nous semble que c'est aussi oublier la valeur d'une entrée de plain-pied dans l'alternance vertueuse entre ce que Schön (1994) appelle la réflexion en action et sur l'action (alors qu'il est généralement admis que c'est justement dans ce type de dynamique que s'est développé, chez l'enseignant portant le nom d'expert, la capacité à une récurrente virtuosité). Plus largement, elle empêche d'appréhender la dynamique de l'activité-improvisation comme une source privilégiée conduisant à l'opérationnalité d'(inter)action, en ce qu'elle est une performance (Lobman, 2011), corrélative de transformations, de (ré)générations (et de motivation).

Certaines professions semblent, de longue date, avoir défendu, voire provoqué, cette possibilité de l'émergence de l'activité-improvisation, dès l'aube du développement professionnel. L'exemple de la formation au métier du barreau, décrite par Paignon (1846) rappelle les procédés parmi les plus actifs d'accompagnement à la construction de savoirfaire. Pour reprendre le parallèle avec la construction du savoir nager, des situations proposant d'emblée de "se jeter à l'eau », dans une interaction avec le milieu, l'exercice consistant d'abord et très tôt en une interaction située, garantissant à la fois la sécurité objective et un degré suffisant d'insécurité subjective. 

l'expérimentation de l'activité-improvisation en interaction en classe, c'est reconnaître la capabilité du débutant, c'est lui garantir la possibilité d'aller à l'aveuglette, à la devinette, c'est substituer la logique de l'homme de progrès à celle de l'expert, c'est-à-dire celle de «l'homme qui marche, qui va voir, qui expérimente, change sa pratique, vérifie son savoir, et ainsi sans fin. » (Rancière, 1987, p. 194) et non celle de l'homme qui est arrivé (même si c'est en un lieu où il est roi de l'adaptation), qui sait faire et qui est enfin prêt à enseigner aux autres ce lieu où il est arrivé.

Dans certains cas cette offre existe déjà, par le moyen des stages. Les questions qui se posent sont alors : celle du moment de leur arrivée en formation, celle de leur volume, de leur fréquence, celle de leur articulation effective avec d'autres formats d'accompagnement au développement professionnel (et à la co-construction de soi), celle enfin, de leur degré de difficulté en relation avec ce que peut le futur ou le néoenseignant. Sur ces interrogations, notre travail ne permet d'apporter qu'un éclairage bien modeste. Nous ne reviendrons pas sur le fait que, comme le défendent certains auteurs, une classe est un environnement dynamique (Rogalski, 2003) qui présente ses risques (Ria, 2009, 2012), un environnement qui, à l'instar de ceux des activités physiques de pleine nature, est potentiellement perturbant, voire dangereux, et qui demande au débutant (comme au confirmé) de la prudence quand il se destine à opérer sa rencontre. Nous souhaiterions simplement souligner que, suivant nos résultats, c'est un niveau d'aisance suffisant (ce qui n'est pas la même chose qu'un niveau d'aisance maximal) qui, chez les néo-enseignants, participe du fait d'oser se lancer dans une interaction en classe. Ils vont donc dans le sens d'un engagement sans délai, de l'enseignant débutant dans une activité d'interaction en classe (sous condition de la garantie d'une sécurité objective suffisante des élèves et de l'enseignant débutant lui-même). Ils nous incitent ainsi, à rejoindre les formats d'enseignement-apprentissage que proposent certaines écoles de musique (par exemple l'école Kaddouch, voir Moindrot, 2012) qui font du jeu musical et de l'improvisation leurs moyens privilégiés d'accompagnement du développement des savoirs et savoir-faire. Pour le dire avec les mots du cadre théorique du cours d'action, la difficulté, côté formation, est ici de contribuer au fait que l'enseignant débutant participe à la viabilité de son couplage à son environnement, notamment par une bascule dans une activité-improvisation, autrement dit par une bascule dans une performance (Lobman, 2011). La gageure réside donc, côté formation, dans le fait de mettre en place les conditions d'un couplage dont le potentiel développemental est élevé. Nous avons vu que l'estimation improvisationnelle disparaît au-delà d'un certain seuil de perturbation, et avec elle le sens du métier et de ce qui s'y joue.

\subsection{Accompagner le déploiement du potentiel de l'activité-improvisation}

85 Nous ne reviendrons pas ici sur les dispositifs présentés dans la revue de littérature proposée par Azéma (2015), qui consistent par exemple: a) en la participation des étudiants ou stagiaires à des ateliers d'improvisation théâtrale (Archieri, 2013 ; Lobman, 2011 ; Pelletier \& Jutras, 2008; Shem-Tov, 2011), b) en la mise en place de situations d'analyse de pratique sur la base de vidéos de classe (Leblanc, 2012; Weisemes \& Wang, 2010) ou d'autres, engageant réflexion sur l'action et prise de conscience (Perrenoud, 1994, 2001a, 2001 b), c) ou encore de situationsoriginales de formation en didactique 
(Maheux \& Lajoie, 2010 ; Towers \& Martin, 2009), ou d) ou d'autres encore, s'apparentant à des jam-sessions (Humphreys \& Hyland, 2002). Nous souhaiterions, sur la base de nos résultats, discuter et/ou mettre en valeur certains facteurs dont il nous semble qu'ils pourraient contribuer à l'émergence et au développement du pouvoir de l'activitéimprovisation.

\subsubsection{Accompagner le développement du courage et soutenir la confiance}

Nos résultats nous montrent que l'activité-improvisation elle-même contribue, dans une large mesure, à cette satisfaction, à ce sens, à l'aisance de l'interaction en classe, avec parfois des rires, mais non sans sueur et avec aussi quelques douleurs. L'aventure de l'interaction en classe, condition d'une possible activité-improvisation, demande donc du courage.

C'est en un sens, une bonne nouvelle que la réalité de l'insécurité de l'interaction en classe, et le courage qu'elle implique. En effet, si « le sentiment de sécurité apporté par l'expérience favorise l'improvisation en permettant de se lancer en avant sans l'angoisse du vide, extrapolant des techniques maîtrisées aux techniques inconnues (...) il gêne l'improvisation en tendant à conserver dans son aire ce qui serait tenté par le risque aventureux » (De Raymond, 1980, p.121), et le risque devient alors celui de tourner en rond dans ses propres clichés.

Sans ce courage, il n'y a pas d'entrée dans le jeu, pas de commencement (De Raymond, 1980), pas de «saut dans l'inconnu » (Laborde, 2005), alors toute activité-improvisation est interdite. Les auteurs étudiant l'improvisation en tant que praxis sont nombreux à se retrouver sur ce point. L'improvisation à la fois implique le courage et «conduit à l'audace, car à refuser le risque de la défaite, on court celui de la sclérose: du professionnalisme selon les règles de l'académie » (De Raymond, 1980, p. 200).

Ce courage et l'activité-improvisation qui peut en résulter, supposent sans doute de triompher de son amour propre (Paignon, 1846), d'apprendre à se vaincre et d'accepter le jugement de l'autre (Rancière, 1987) - élèves, collègues, etc. Nos résultats montrent que ce courage de commencer suppose aussi, une aisance suffisante de l'enseignant débutant (avec le contenu d'enseignement et/ou avec le lieu de travail et son matériel et/ou avec les autres, etc.), corrélative d'une confiance a priori. Bailey (2011, p.131) évoque l'enseignement de John Stevens (praticien et organisateur de "musiques free») consistant à donner aux gens avec qui il travaille, assez de confiance pour qu'ils essaient de faire ce dont ils ont envie avant même de savoir comment.

En relation avec nos résultats et selon notre propre expérience de formation, nous retenons deux réflexions, dont il nous semble qu'elles sont propres à aller dans le sens d'un déploiement de la confiance et du courage :

- Notons tout d'abord que si, comme le souligne Miles Davis, on improvise toujours au-dessus de ses moyens (Hervé, 2012) (sous-entendu, à son niveau, ou en dessous, on n'improvise pas, mais on ressasse des clichés), l'énorme bagage dont il est fréquemment avancé que sans lui il n'y a pas d'improvisation possible se tourne en mythe davantage qu'il ne demeure une nécessité. Retenons que les enfants, dans un apprentissage «du coin de la rue » (McLaren cité par Baker-Sennett \& Matusov, 1997), font avec et au-delà des moyens de leur bord. Dans le dernier cas, l'avantage de la situation réside dans la possibilité toujours présente de quitter le jeu. C'est, au final, surtout le fait que l'acteur ait la possibilité de jouer sur le juste niveau de difficulté du couplage acteur/environnement qui semble déterminant. Cette 
possibilité donnant entre autres la possibilité à l'acteur d'abandonner pour un temps l'interaction (pour y revenir, une fois regonflé - un peu à la manière d'un surfeur qui se pose 30 minutes après s'être fait secouer, pour, une fois à nouveau d'attaque, après un temps de récupération et de « réflexion », revenir « à la charge »);

- Soulignons ensuite avec De Raymond (1980, p. 59) que «l'incapacité à improviser tient à un contexte psychologique et social (...) Cependant blocages psychologiques et congères de l'histoire personnelle sur lesquels a prise la rationalité scientifique ne sauraient passer pour un destin auquel certains seraient voués, loin des heureux doués pour un art alors divinisé (...) Inversement, la pratique de l'improvisation contribue à dissoudre les blocages psychologiques qui l'entravent (...) apprendre à improviser est apprendre à revivre ». De notre côté, nous avons avancé que si se jeter à l'eau implique une confiance suffisante, l'activité-improvisation donne de la confiance.

\subsubsection{Accompagner le développement de l'attention}

91 Nos résultats ont mis en exergue le régime attentionnel et interactionnel spécifique de l'activité-improvisation. Tout à la fois, au cours de l'activité-improvisation, le régime attentionnel et interactionnel monte d'un cran (on peut donc penser qu'elle développe et/ou renforce les dispositions corrélatives) et il apparaît qu'il participe à son opérationnalité. Nous revenons ici sur la question de l'attention (fréquemment associée à celui de présence, de vigilance ou d'écoute), en prenant pour acquis qu'elle est chaînée à celle d'interaction.

Cette question nous conduit à rejoindre le point de vue de nombre d'auteurs sur ce en quoi elle consiste et sur son rôle clé (e.g. Barker \& Borko, 2011 ; Lobman, 2005 ; Pelletier \& Jutras, 2008). Selon Barker et Borko (2011), il faut comprendre la présence-attention comme une «conscience en alerte» et multidirectionnelle à soi, aux apprenants (individuellement et collectivement), au contenu de l'enseignement, au contexte (ce qui va dans le sens d'une co-dépendance des régimes attentionnel et interactionnel). Ils notent par ailleurs qu'elle implique une ouverture à un contrôle partagé de la situation. Nous rejoignons Lopez de Maturana Luna (2010) lorsqu'elle souligne que l'attention a à voir avec des signes, notamment non verbaux, subtils (même si nous pensons qu'elle ne se porte pas sur eux, mais plutôt, que la dynamique du couplage acteur/environnement permet leur activité). Ce qui nous intéresse particulièrement ici, c'est le point de vue de Pelletier et Jutras (2008, p. 210) selon lequel le développement de la vigilance (et de la rapidité de réflexion en action) "pourrait compenser, dans certains cas, le manque d'expérience des stagiaires ou des enseignants novices ».

Pour Pelletier et Jutras (Ibid.), l'entraînement à l'improvisation théâtrale (comme l'improvisation en classe) contribue à ce type de développement. Il est intéressant de noter que c'est un type d'expérience qui n'appelle aucune compétence didactique (ou son développement). On peut donc penser que, si la capacité de présence-attention implique des bases de connaissances étendues, approfondies, flexibles (Barker \& Borko, 2011), il est des dispositions à agir qui les compensent, voire contribuent à leur construction (rappelons que le savoir-faire didactique se construit aussi en interaction). Ceci nous amène à souligner que les dynamiques récursives désarçonnent les questions du type : faut-il improviser pour savoir ou savoir pour improviser ? Faut-il connaître pour être attentif ou être attentif pour connaître? qui obligent à trancher entre l'un de leurs termes. Elles rappellent que c'est en appréhendant les deux termes, donc en s'inscrivant dans des dynamiques en boucles de co-dépendance, que semble se jouer tout processus 
d'individuation. Nos résultats nous invitent à redorer le blason de l'arc de la boucle consistant dans l'acte, peut-être imparfait, mais développemental.

\subsubsection{Une certaine façon de considérer la préparation}

Qu'elle soit recherchée ou non par les néo-enseignants, l'activité-improvisation ne se décrète pas, ne se contrôle pas. Elle émerge, s'invite à la table. On pourrait considérer qu'il n'y a pas lieu, dans le cadre de notre recherche, d'aborder la question de la préparation. Pourtant ce que nous avons observé et compris de l'activité-improvisation nous incite à le faire, motivés par le fait qu'il est vraisemblable que, comme le souligne De Raymond (1980, p. 121):

«Certaines préparations étouffent tout tonus créatif, mais l'absence de préparation

laisse démuni devant l'infinie variété des situations complexes ».

Nous souhaitons notamment ici, tenter de remettre en question cette question de la préparation qui, telle que conçue en enseignement scolaire pour ce qui concerne les néoenseignants du premier et du second degré, semble, sous l'influence d'une culture de la science appliquée et de l'efficacité bureaucratique (Schön, 1994), avoir pris le sens restrictif de la trace écrite d'une planification programmatique de séance. Or se préparer, dans le sens de : se disposer à son émergence et à son mouvement, nous semble appeler une autre considération de la préparation.

6 Au-delà de la commande institutionnelle à laquelle certains enseignants du premier et du second degré sont sommés de répondre, nos résultats (en négatif au sens photographique) nous ont donné à comprendre qu'elle consiste dans une large mesure, chez les néo-enseignants, en l'atteinte d'un seuil d'aisance et de confiance suffisant. Un seuil qui donne du cœur au ventre, pour aller s'aventurer en interaction en classe. Nous avons évoqué le fait que les néo-enseignants semblaient comprendre très tôt qu'il ne saurait y avoir de contrôle a priori de la situation, qui souvent leur échappe.

Nous souhaiterions réaffirmer ici la différence, selon nous nécessaire, qu'il convient de faire (suivant Suchman, 1990) entre planification programmatique et préparation ressource. La préparation comme ressource (gain de confiance, source de courage, inspiratrice, participant de l'orientation de l'interaction, sans la déterminer) est, selon nous, un atout. Elle déborde largement les traces éventuelles qui seront laissées sur un papier ou dans un fichier numérique. Il semble que l'accompagnement au développement professionnel ait à gagner à soutenir la construction d'un type personnel de préparation, qui soit tout sauf une entité verrouillée. Qui peut éventuellement comprendre de multiples formes de transcriptions (écriture, mais aussi dessins ou schémas), dont la mise en forme reste libre, ou, paradoxalement consister en impréparation de traces écrites. De façon à ce que, comme y insiste Carl Whitmer (cité par Bailey, 2011) concernant l'improvisation à l'orgue, dans le cours de l'interaction: a) l'idée reste dans un état de flux, b) l'erreur puisse se révéler positive, c) soient privilégiés l'énergie et l'élan. Schön (1994), relativement à la planification de l'enseignant, parle d'esquisse, de squelette autour duquel il est possible de broder des variations. Pourquoi faudrait-il interdire ce procédé aux enseignants débutants?

98 Si nous nous en tenons à nos résultats, il parait en outre important de faire en sorte que la préparation puisse impliquer et respecter des temps d'imagination active (qui ne se font pas qu'en images), comme si l'enseignant se faisait le scénario d'un film. Cette préparation sensible pouvant donner lieu à des pense-bêtes ou à la préparation d'un 
certain matériel, et se faire ailleurs qu'à son bureau (notamment sur le lieu de la future interaction ou dans des lieux plus improbables).

À une échelle plus générale, la préparation (jamais achevée) consiste en la participation à de multiples cultures (professionnelles, didactiques et autres), pour démultiplier le potentiel inspirateur. Nos résultats nous donnent en effet à comprendre que ce qui émerge lors de l'activité-improvisation est à la fois connu et inconnu, et que ce qui vient à l'acteur, inspire son action, émerge sur la base d'un écho, d'une résonance liée, comme le souligne le cadre théorique du cours d'action, à un air de famille de l'activité et du couplage en cours avec l'activité et un couplage passé. La préparation pourra encore privilégier la récupération physique, le sommeil, garantie dans certains cas d'une capacité attentionnelle optimale. Elle pourra encore consister en un entraînement régulier à la présence attentive, comme évoqué plus haut.

Concluons cette sous-section en redisant et en défendant le fait que l'activitéimprovisation elle-même est préparation (de la suivante). En ce sens qu'elle est l'espacetemps privilégié : a) du développement et de l'affinage des dispositions qui font son opérationnalité : dispositions à accuser le coup, à être attentif, à saisir l'opportunité, à masquer ses émotions, à lâcher prise, à accompagner un mouvement qui dépasse, à transgresser (pour sauver l'essentiel), b) de (re)découvertes. «J'arrive à les voir [les élèves, au cours d'une préparation de séance], parce que je les ai vus » nous dit Cindy, professeure des écoles stagiaire.

\subsubsection{Quelle place et quel rôle du formateur?}

101 Selon nous, il serait dommageable que des formateurs se fassent un jour les détenteurs de l'explication et de l'enseignement de ce qu'ils pourraient appeler l'Improvisation professionnelle. Ce serait faire exister de fait, un groupe d'ignorants ou pire, d'incapables; ce serait leur faire croire que c'est une activité réservée, ou bien une activité à laquelle il s'agit de se conformer. Ce serait encore, sans doute, l'indicateur d'une tentative de faire rentrer dans le rang une activité qui par nature, échappe. De Raymond (1980, p. 64) invite à se défier «de ceux qui prétendent monopoliser les règles d'apprentissage de l'improvisation pour mieux la circonscrire: ils la réduisent ou ils l'annexent (...) ils la supposent constituée déjà comme un savoir et une orthodoxie par rapport à une ignorance et une hérésie ».

102 Ce que (peut-être) peut en revanche le «formateur » c'est, en s'intéressant à lui/elle, accompagner chez l'autre l'émergence de l'activité-improvisation. Notons qu'il n'y a pas, selon nous, de position confortable d'un côté et de position inconfortable de l'autre, accompagner le courage de la prise de risque demandant à l'accompagnant d'en prendre lui-même.

103 Il peut être intéressant, dans le cadre d'une réflexion consistant à considérer l'accompagnement de l'émergence de l'activité-improvisation, de revenir sur celles auxquelles Rogers (2005) avait abouti au moment d'un bilan qu'il avait été amené à faire au sujet de l'enseignement. Il en était arrivé à penser qu'il ne pouvait enseigner à quelqu'un d'autre à enseigner, «que ce qui peut être enseigné à une autre personne est relativement sans utilité et n'a que peu ou point d'influence sur son comportement. (...) Que les seules connaissances qui influencent réellement l'individu sont celles qu'il découvre lui-même et qu'il s'approprie » (Ibid., p. 185). Il en était arrivé à la conclusion que ce qui l'intéressait plutôt était d'apprendre, et que la meilleure façon de le faire 
consistait à «comprendre comment une autre personne conçoit et éprouve sa propre expérience » (Ibid., p. 186). Il tire de cette conclusion, et de son expérience de thérapeute, des conditions propres à servir l'enseignement-apprentissage (Ibid., p. 185-186) : a) le fait de partir de la confrontation à un problème que l'étudiant est avide de résoudre, b) l'authenticité de l'enseignant (impliquant qu'il soit véritablement ce qu'il est, sans façade) qui permet d'être pleinement conscient et de vivre l'expérience d'autrui, c) l'acceptation et la compréhension, incluant: la capacité de l'enseignant à une considération positive inconditionnelle de l'étudiant (entendue comme une attention chaleureuse, qui ne demande aucune gratification personnelle, et impliquant d'accepter les sentiments négatifs), la capacité de l'enseignant à une compréhension "empathique " (entendue comme la capacité à éprouver une compréhension exacte du monde de l'autre comme s'il était perçu de l'intérieur), le fait que l'étudiant éprouve ou perçoive quelque chose de la congruence, de l'acceptation ou de l'empathie manifestée, d) la mise à disposition de ressources (et non l'imposition de ressources), e) la confiance fondamentale dans la tendance des étudiants à s'affirmer eux-mêmes.

\section{Conclusion}

En conclusion de cet article, nous souhaitons souligner que le point de vue proposé par cette recherche permet de reconsidérer le fait de réserver le qualificatif d'improvisationnel au fonctionnement de l'enseignant dit expert, ou chevronné. Le fonctionnement improvisationnel (Tochon, 1993) est généralement considéré comme la capacité à s'adapter à toutes sortes de situations - qui se fait jour à la suite de nombreuses années de pratique, donc sur la base d'un large répertoire de routines. Les néoenseignants en sont généralement tenus pour peu ou pas capables. Or, selon nos résultats, l'activité-improvisation n'est pas d'abord une question de capabilité préalable, mais un incontournable du chemin conduisant au professionnalisme, et permettant de rester professionnel. Ce que nos résultats mettent en avant, c'est que l'activité-improvisation ne se confond pas avec une simple activité d'adaptation, mais qu'elle correspond à une adaptation doublée d'une invention (Bailey, 2011), y compris de soi. Encore une fois, il semble bel et bien que l'on improvise toujours au-dessus de ses moyens (Miles Davis, in Hervé, 2012) du moment.

Cette reconsidération nous semble avoir quelque pertinence, dans le sens où le point de vue consistant à confondre improvisation et adaptation, en réservant cette capacité à l'enseignant dit expert, présente selon nous, au moins un écueil. Il n'est pas question de défendre que les années de pratique ne soient en rien dans un possible gain d'opérationnalité pratique (en quantité et qualité en n'oubliant toutefois pas que certaines études ont montré que le temps de pratique du métier peut aussi correspondre à un effritement ou à une baisse de cette opérationnalité, e.g. Durand, 1996). Le bémol est ailleurs : cette considération fait exister et creuse une différence entre deux mondes, dans une logique verticale du haut et du bas et dans une logique de séparation exclusive ou de dénigrement de l'un des deux mondes (le monde d'en bas, étant généralement réservé aux novices). Cette conception usuelle de l'improvisation en enseignement scolaire comme activité réservée aux virtuoses, suite à des années de gammes et/ou suite à des années d'interactions en classe, nous semble porter en germe, le risque d'une sorte d'infantilisation voire celui d'un "abrutissement» (Rancière, 1987). Alors qu'on peut 
considérer qu'il faut improviser non seulement pour devenir un professionnel, mais aussi, pour le rester.

Sur le plan de la formation, ce sont donc les écarts et non les différences (Jullien, 2016 ${ }^{18}$ ) de dispositions à agir entre néo-enseignants et formateurs (ou autres enseignants dits chevronnés) qu'il peut être intéressant d'appréhender, mais sans que la valeur de cet écart ne soit positivement considérée qu'en direction des seconds, et en mettant aussi en avant ce que les deux ont en commun. Cela, sans perdre de vue le souci d'une exigence de qualité des interactions d'enseignement-apprentissage, dont il peut être attendu qu'elle rime avec la multiplication des co-(ré)-générations heureuses.

En un sens, sans doute est-ce une bonne nouvelle que d'entendre des néo-enseignants dire qu'ils improvisent, et qu'ils nous aident à comprendre comment.

\section{BIBLIOGRAPHIE}

Arborio, A.-M., \& Fournier, P. (1999). L'enquête et ses méthodes : l'observation directe. Paris : Nathan.

Archieri, C. (2013). La construction de l'expérience par le théâtre. Thèse de doctorat non publiée. Université de Bretagne Ouest, Brest.

Azéma, G. (2015). L'improvisation selon les enseignants entrant dans le métier. Une approche en anthropologie cognitive. Thèse de doctorat non publiée. Université Montpellier 3.

Bailey, D. (2011). L'improvisation. Sa nature et sa pratique dans la musique (3e éd.) (I. Leymarie, trad.). Paris : Outre Mesure. (Ouvrage original publié en 1980 sous le titre Improvisation: Its Nature and Practice in Music. Ashbourne : Moorland Publishing).

Baker-Sennett, J., \& Matusov, E. (1997). School “performances”: Improvisational processes in development and education. In R. K. Sawyer (Ed.), Creativity in performance (p. 197-212). Greenwich, CT : Ablex Publishing Company.

Barker, L., \& Borko, H. (2011). Conclusion: Presence and the art of improvisational teaching. In R. K. Sawyer (Ed.), Structure and improvisation in creative teaching (p. 279-298). Cambridge : Cambridge University Press.

Beaud, S., \& Weber, F. (2010). Guide de l'enquête de terrain (4éd.). Paris : La Découverte. (Ouvrage publié en 1997).

Becker, H.S. (1970). Social Work. Method and substance. New York : Rouledge.

Billeter, J.-F. (2010). Leçons sur Tchouang-Tseu. Paris : Allia.

Borko, H., \& Livingston, C. (1989). Cognition and improvisation: Differences in mathematics instructions by expert and novice teachers. American Educational Researcher Journal, 26(4), 473-498.

Brown, M., \& Edelson, D. (2003). Teaching as design. The center for Learning Technologies in Urban Schools: LeTUs Report Series.

Bucheton, D., \& Soulé, Y. (2009). Les gestes professionnels et le jeu des postures de l'enseignant dans la classe : Un multi-agenda de préoccupations enchâssées. Éducation et didactique, 3(3), 29-48. 
Chapoulie, J.-M. (1985). Préface. In H., S. Becker, Outsiders. Étude sociologique de la déviance (p. 9-21). Paris : Métaillé

Chauviré, C. (2002). Dispositions ou capacités ? La philosophie sociale de Wittgenstein. In

C. Chauviré \& A. Ogien (Eds.). La régularité. Habitude, disposition et savoir-faire dans l'explication de l'action (p. 25-48). Paris : EHESS.

De Certeau, M. (1990). L'invention du quotidien 1. Arts de faire. Paris : Gallimard.

De Raymond, J.-F. (1980). L'improvisation. Paris : Vrin.

DeZutter, S. (2011). Professional improvisation and teacher education: Opening conversation. In R. K. Sawyer (Ed.), Structure and improvisation in creative teaching (p. 27-50). Cambridge : Cambridge University Press.

Durand, M. (1996). L'enseignement en milieu scolaire. Paris : PUF.

Durand, M. (2008). Un programme de recherché technologique en formation des adultes : une approche enactive de l'activité humaine et l'accompagnement de son apprentissage/ développement. Éducation \& Didactique, 2(3), 97-121.

Durand, M., \& Perrin, N. (2014). Dispositions et transformation de l'activité d'une professionnelle débutante. In A. Muller \& I. Plazaola Giger (Eds.), Dispositions à agir, travail et formation (p. 12-31). Toulouse : Octarès.

Durand, M., \& Veyrunes, P. (2005). L'analyse de l'activité des enseignants dans le cadre d'un programme d'ergo-formation. Les Dossiers de Sciences de l'Éducation, 14, 47-60.

Erickson, F. (1982). Classroom discourse as improvisation : Relationship between academic task structure and social participation structure in lessons. In L.C. Wilkinson (Ed.), Communicating in the Classroom (pp. 153-181). New York: Academic Press.

Erickson, F. (2011). Taking advantage of structure to improvise in instruction: Examples from elementary school classrooms. In R. K. Sawyer (Ed.), Structure and improvisation in creative teaching (p. 113-132). Cambridge : Cambridge University Press.

Fournier, J. (2011). Productive improvisation and collective creativity: Lessons from the dance studio. In R. K. Sawyer (Ed.), Structure and improvisation in creative teaching (p. 184-206). Cambridge : Cambridge University Press.

Gagnon, R. (2011). L'improvisation théâtrale au service de l'expression orale et écrite et de son enseignement. Revue Suisse des Sciences de l'Éducation, 33(2), 251-265.

Gauthier, C., Desbiens, J.-F., Malo, A., Martineau, S., \& Simard, D. (1997). Pour une théorie de la pédagogie. Recherches contemporaines sur le savoir des enseignants. Bruxelles : De Boeck Université. Geay, B. (2010). Les néo-enseignants face à l'utilitarisme. Actes de la recherche en sciences sociales, 184, 72-89.

Gershon, W. (2006). Collective improvisation: A theoretical lens for classroom observation. Journal of curriculum and pedagogy, 3(1), 104-135.

Hervé, A. (2012). Retour sur l'improvisation. L'éducation musicale, 575, 23-25.

Huberman, M. (1993). Enseignement et professionnalisme : Des liens toujours fragiles. Revue des sciences de l'éducation, 19(1), 77-85.

Humphreys, M., \& Hyland, T (2002). Theory, practice and performance in teaching: Professionalism, intuition, and jazz. Educational Studies, 28(1), 5-15.

Jullien, F. (2016). Iln'y a pas d'identité culturelle. Paris : L'Herne. 
Kelly, G. J., Brown, C., \& Crawford, T. (2000). Experiments, contingencies, and curriculum: Providing opportunities for learning through improvisation in science teaching. Science Education, 84, 624-657.

Laborde, D. (2005). La mémoire de l'instant. Les improvisations chantées du bertsulari basque. Bayonne : elkar.

Lahire, B. (2006). Culture. In S. Mesure \& P. Savidan (Eds.), Le dictionnaire des sciences humaines (p. 232-234). Paris : PUF.

Lahire, B. (2011). L'homme pluriel. Les ressorts de l'action. Paris : Fayard. (Ouvrage publié en 2001).

Lantheaume, F. (2008). De la professionnalisation à l'activité : nouveaux regards sur le travail enseignants. Recherche et Formation, 57, 9-22.

Laplantine, F. (2006). La description ethnographique. Paris : Armand Colin.

Leblanc, S. (2012). Conception d'environnements vidéo numériques de formation. Développement d'un programme de recherche technologique centré sur l'activité dans le domaine de l'éducation. Note de synthèse pour l'Habilitation à Diriger des Recherches non publiée. Université Paul Valéry Montpellier 3.

Lobman, C. (2003). What should we create today? Improvisation teaching in play-based classrooms. Early Years, 23(2), 131-142.

Lobman, C. (2005). Yes and: The uses of improvisation for early childhood teacher development. Journal of Early Childhood Teacher Education, 26(3), 305-319.

Lobman, C., \& Lundquist, M. (2007). Unscripted learning: Using improve activities across the K-8 curriculum. New York: Teachers College Press.

Lobman, C. (2011). Improvising within the system: Creative new teacher performances in innercity schools. In R. K. Sawyer (Ed.), Structure and improvisation in creative teaching (p. 73-93). Cambridge : Cambridge University Press.

Lopez de Maturana Luna, S. (2010). Las complejidades emergentes en las historias de vida de los "buenos profesores". Polis, 25, 1-11.

Mabilon-Bonfils, B., \& Zoïa, G. (2014). La laïcité au risque de l'autre. La Tour d'Aigues : L'Aube.

Maheux, J.-F., \& Lajoie, C. (2010). On improvisation in teaching and teacher education. Complicity: An International Journal of Complexity and Education, 8(2), 86-92.

Maroy, C., \& Cattonar, B. (2002). Professionnalisation ou déprofessionnalisation des enseignants? Le cas de la communauté française de Belgique. Les Cahiers de recherche du GRISEF, 18, 1-26.

Moindrot, G. (2012). L'improvisation : A la recherche du musicien intérieur. L'éducation musicale, 575, 26-29.

Muller, A. (2014). Conclusion générale : retour sur la conception des dispositions développée dans cet ouvrage. In A. Muller \& I. Plazaola Giger (Eds.), Dispositions à agir, travail et formation (p. 189-195). Toulouse : Octarès.

Paignon, E. (1846). Éloquence et improvisation. Art de la parole oratoire au barreau, à la tribune, à la chaire. Paris : Cotillon.

Pelletier, J.-P., \& Jutras, F. (2008). Les composantes de l'entraînement à l'improvisation active dans la gestion des imprévus en salle de classe du niveau secondaire. McGill Journal of Education, 43 (2), 187-211. 
Perez-Roux, T., \& Lanéelle, X. (2015). Pluralité des ressources pour l'action dans les processus de transition formation-emploi chez les enseignants du secondaire. Les Sciences de l'Éducation pour l'ère nouvelle, 48(4), 17-40.

Perrenoud, P. (1994). La pratique pédagogique entre l'improvisation réglée et le bricolage. In P. Perrenoud (Ed.), La formation entre théorie et pratique (p. 21-41). Paris : L'Harmattan. (Article initialement publié in Éducation et Recherche, 1983, 2, 198-212).

Perrenoud, P. (1995). Dix non-dits ou la face cachée du métier d'enseignant. Recherche et Formation, 20, 107-124.

Perrenoud, P. (2001a). De la pratique réflexive au travail sur l'habitus. Recherche et Formation, 36, 131-162.

Perrenoud, P. (2001b). Le travail sur l'habitus dans la formation des enseignants. Analyse des pratiques et prise de conscience. In L. Paquay, M. Altet, E. Charlier, \& P. Perrenoud (Eds), Former des enseignants professionnels. Quelles stratégies? Quelles compétences? (p. 181-207) (3éd.). Paris : De Boeck Université.

Perrin, N., Theureau, J., Menu, J., \& Durand, M. (2011). SIDE-CAR : un outil numérique d'aide à l'analyse de l'activité par rétrodiction. Exploitation selon le cadre théorique du « cours d'action ». Recherches qualitatives, 30(2), 148-174.

Piot, T. (2008). La construction des compétences pour enseigner. McGill Journal of Education, 43(2), 95-110.

Rancière, J. (1987). Le maître ignorant. Cinq leçons sur l'émancipation intellectuelle. Paris : Fayard. Rayou, P., \& Van Zanten, A. (2004). Les nouveaux enseignants. Changeront-ils l'école ? Paris : Fayard. Ria, L. (2009). Les « passages à risques » des enseignants débutants en milieu difficile au cœur du développement de leur activité professionnelle. Colloque international de la CDIUFM : Développement professionnel des enseignants ». Rouen (hal-00804097, version 1, 24 mars 2013).

Ria, L. (2012). Variation des dispositions à agir des enseignants débutants du secondaire : Entre croyances et compromis provisoires. In P. Guibert \& P. Périer (Eds.), La socialisation professionnelle des enseignants du secondaire (p. 107-125). Paris : PUF.

Ria, L. (2014). Dynamique de transformation des dispositions à agir des enseignants débutants : un processus d'autoformation et un enjeu de formation. In A. Muller \& I. Plazaola Giger (Eds.), Dispositions à agir, travail et formation (p. 77-88). Toulouse : Octarès.

Rogalski, J. (2003). Y a-t-il un pilote dans la classe ? Une analyse de l'activité de l'enseignant comme gestion d'un environnement dynamique ouvert. Recherches en Didactique des Mathématiques, 23(3), 343-388.

Rogers, C. R. (2005). Le développement de la personne (E. L. Herbert, trad.). Paris : DunodInterEditions. (Ouvrage original publié en 1961 sous le titre On becoming a Person. Boston : Houghton Mifflin Company).

Sartre, J.-P. (2005). L’imaginaire. Paris : Gallimard. (Ouvrage publié en 1940).

Sawyer, R. K. (2004). Creative teaching: Collaborative discussion as disciplined improvisation. Educational Researcher, 33(2), 12-20.

Sawyer, R. K. (2011). What makes good teacher great? The artfull balance of structure and improvisation. In R. K. Sawyer (Ed.), Structure and improvisation in creative teaching (p. 1-24). Cambridge : Cambridge University Press. 
Schön, D. A. (1994). Le praticien réflexif. A la recherché du savoir caché dans l'agir professionnel (J. Heynemand et D. Gagnon, trad.). Montréal : Les Éditions Logiques. (Ouvrage original publié en 1983 sous le titre The Reflective Practitioner. New York : Basic Books).

Shem-Tov, N. (2011). Improvisation teaching as mode of knowing. Journal of Aesthetic Education, 45 (3), 103-113.

Simondon, G. (2013). L'individuation à la lumière des notions de forme et d'information. Grenoble : Millon. (Texte original, 1958).

Suchman, L. (1990). Plans d'action. Problèmes de représentation de la pratique en sciences cognitives. In P. Pharo \& L. Quéré (Eds.). Les formes de l'action. Sémantique et sociologie (p. 149-170). Paris : EHESS.

Theureau, J. (2004). Le cours d'action : Méthode élémentaire. Toulouse : Octarès.

Theureau, J. (2006). Le cours d'action : Méthode développée. Toulouse : Octarès.

Theureau, J. (2010). Les entretiens d'autoconfrontation et de remise en situation par les traces matérielles et le programme de recherche " cours d'action ». Revue d'anthropologie des connaissances, 4(2), 287-322.

Theureau J. (2011). L'observatoire des cours d'action, des cours de vie relatifs à une pratique et de leurs articulations collectives. In G. Le Meur \& M. Hatano (Eds.) Approches pour l'analyse des activités (p. 23-76). Paris : L'Harmattan.

Theureau, J. (2015). Le cours d'action : L'enaction \& l'expérience. Toulouse : Octarès.

Tochon, F. V. (1989). Peut-on former les enseignants novices à la réflexion des experts ? Recherche et Formation, 5, 25-38.

Tochon, F. V. (1992). Trois épistémologies du bon enseignant. Revue des sciences de l'éducation, 18 (2), 181-197.

Tochon, F. V. (1993). Le fonctionnement « improvisationnel » de l'enseignant expert. Revue des sciences de l'éducation, 19(3), 437-461.

Towers, J., \& Martin, L. (2009). The emergence of a "better" idea: Preservice teachers growing understanding of mathematics for teaching. For the learning of mathematics, 29(3), 44-48.

Wiesemes, R., \& Wang, R. (2010). Video conferencing for opening classroom doors in initial teacher education: Sociocultural processes of mimicking and improvisation. International Journal of Media, Technology and Longlife Learning, 1(6), 28-42.

Wittorski, R., \& Briquet-Duhazé, S. (2009). Dynamique de professionnalisation / développement professionnel d'enseignants : étude comparative entre les premier et second degrés. In R. Goigoux, L. Ria, \& M.-C. Toczek-Capelle (Eds.), Les parcours de formation des enseignants débutants (p. 45-60). Clermont-Ferrand : Presses universitaires Blaise Pascal.

Yinger, R. J. (1987a). By the seat of your pants: An inquiry into improvisation and teaching. Paper presented at the annual meeting of the American Educational Research Association, April, Washington, DC.

Yinger, R. J. (1987b). Learning the language of practice. Curriculum Inquiry, 17(3), 293-318.

Zoïa, G., \& Visier, L. (2013). Professionnalisation/Déprofessionnaliosation. Une comparaison entre enseignants et médecins. Recherche et Formation, 72(1), 41-56. 
ANNEXES

1 - Chronologie et précisions concernant la construction des données. Une série de méthodes assemblées

Quand?

Selon les opportunités de visites ou de rencontres de stagiaires.

Qui ?

Différents professeur.e.s des écoles-stagiaires dans le cadre de notre travail de formateur.

Comment?

Observations directes. Entretiens opportunistes.

Où ?

École ou faculté. 


\section{Quand?}

Entre le 18-09-2013 et le 16-01-2014.

Qui?

23 néo-enseignants (stagiaires ou titulaires première année).

Femmes et des hommes, enseignants dans le premier degré (cycles 1 et 2), le second degré

(collège) et l'université, dans des secteurs géographiques et socioculturels diversifiés (Hérault, Isère, banlieue parisienne). La présence d'un nombre important de variables [âge, sexe, origine sociale, expériences sportives et artistiques, expériences préprofessionnelles, types de formation, situation parentale, niveaux de classe enseignés, matière(s) enseigné(e)s, temps effectif d'enseignement en équivalent temps plein, implantation socioculturelle de l'établissement] donne une bonne représentativité de la population néo-enseignante et est susceptible de mieux faire ressortir ce qui vaut pour elle au-delà des particularismes et des couplages micro-situationnelles spécifiques.

5 néo-enseignants ont, au final, de 2 à 4,5 ans équivalent temps plein d'expérience (dont 4 enseignants universitaires, leur service année étant de 192 heures équivalent TD (pour Travail Dirigé). Ceci se comprend du fait que ces enseignants sont titulaires première année et qu'ils ont enseigné au cours de leur thèse durant maximum $1 / 4$ temps/an en moyenne). Ce point est pondéré par le fait que les expériences auxquelles ont fait référence les participants ont justement été vécues alors qu'ils étaient doctorant-moniteurs. La moyenne d'âge des enquêtés est de 29,5 ans, avec un écart type de 5,87 ans. $35 \%$ des professeurs des écoles enquêtés travaillent en zone dite « difficile ». $100 \%$ des enseignants de collège enquêtés travaillent en établissements dits difficiles.

\section{Comment?}

Entretiens ethnographiques (Beaud \& Weber, 2010).

Les entretiens ethnographiques peuvent déboucher sur diverses verbalisations. Nous avons été attentifs à produire des descriptions commentées qui prennent appui sur des situations personnelles précises, incarnées, vécues, accompagnées de sentiments, etc. Nous avons fait en sorte de repérer et/ou d'éviter : a) les verbalisations plus ou moins " complices de soi » (Theureau, 2015), b) les jugements de soi, c) les réponses de pures conventions, parfois pour faire plaisir, d) les tentatives d'analyses distanciées en mobilisant un savoir théorique sur le propos en cours. Concernant ces entretiens ethnographiques, nous souhaitions rappeler qu'avant et pendant les interactions verbales, le chercheur est présent au corps de l'interviewé, à ses manières, au lieu qu'il a choisi pour effectuer l'entretien. Il s'en imprègne. Pour que porte le jeu de proximité/distance permettant de comprendre le monde voire l'activité de l'autre, la pratique ethnographique demande une forme particulière d'attention (à la fois orientée et flottante) (Laplantine, 2006), à soi (ce que je vis, ce qui m'émeut, ce qui me dérange, me révolte, me surprend) et à l'autre. Cette attention est loin de ne considérer que le discours verbal (Beaud \& Weber, 2010).

où?

Chez les acteurs. Sur le lieu de travail. Dans des bars. 2 entretiens effectués dans notre propre bureau. 


\section{Quand?}

Entre le 25-01-2014 et le 15-04-2014.

Qui?

6 acteurs parmi les 23 . A l'issue de l'entretien ethnographique 1, pour engager une étude qualitative plus poussée, une présentation de la suite de la recherche était faite à l'acteur et une collaboration plus ample lui était proposée. En tenant compte de la lourdeur du protocole à venir (donc du facteur faisabilité), nous avons retenu 6 acteurs volontaires. Nous avons été attentifs à ce qu'ils soient le plus représentatifs possibles de la population ciblée. Un enseignant et une enseignante du premier degré (cycle 1 et 2 ) ; un enseignant de collège ; deux enseignants chercheurs (Nanobiologie et Physique) et une enseignante chercheure (Pharmacie).

\section{Comment?}

Observations participantes. Elles ont été réalisées dans 14 classes : 2 classes de moyenne et grande section de maternelle, 1 classe de Cours Préparatoire ; 2 classes de $6^{\mathrm{e}}, 1$ classe de $3^{\mathrm{e}} ; 1$ classe de licence première année ( $\mathrm{TD}^{19}$ de chimie), 1 classe de licence troisième année de nanobiologie (TD chimie),1 classe de licence troisième année de nanobiologie (CM chimie), 2 classes de licence troisième année de physique (TD électricité), 1 classe de deuxième année d'IUT génie mécanique (TP), 2classes de Licence deuxième année de pharmacie (TP).

La construction du matériau empirique en observation directe a été accompagnée : de notes ethnographiques, de photos et plans (Arborio \& Fournier, 1999 ; Beaud \& Weber, 2010, Laplantine, 2006) et de vidéographies. Nous avons aussi réalisé des micro-entretiens « à chaud » dans la foulée des temps de classe, visant notamment le ciblage par l'acteur, d'épisodes qu'il estimait être « de l'improvisation » - avec précision du degré à partir d'une échelle de Likert -3/ +3. (-3) : Estime ne pas du tout improviser ou vivre une improvisation... $(-2,-1,0,+1,+2) \ldots(+3)$ : Estime très fortement improviser ou vivre une improvisation].

D'autres micro-entretiens « à chaud ", à la fin de la journée de travail, ou des entretiens opportunistes (par exemple lors d'un repas partagé avec l'acteur) ont encore été réalisés. Nous avons par ailleurs renseigné un journal du chercheur, qui visait à distiller notre expérience, et à nous rapprocher de l'activité observée tout en la mettant à distance. Dans ce jeu de proximité/ distance auquel se livre l'ethnographe, en tenant compte des malentendus, des étrangetés, des bizarreries, etc. qui existent pour lui et qui, paradoxalement, peuvent contribuer à mieux comprendre l'autre.

Où ?

Dans les salles de classe.

Quand?

Entre le 25-01-2014 et le 15-04-2014.

Qui?

6 acteurs parmi les 23.

Comment?

Entretiens d'autoconfrontation de premier niveau ou de remise en situation dynamique. Suivant leur richesse estimée, 4 corpus de données ont été analysés en signes. (L'ensemble des données a fait l'objet d'une analyse inductive).

où ?

Sur le lieu de travail. Salle de classe ou bureau de l'acteur. 


\section{Quand?}

Entre le 25-03-2015 et le 24-04-2015.

Qui?

3 acteurs parmi les 6 . Pour lesquels les données construites avaient été analysées en signes.

Comment?

Entretiens d'autoconfrontation de second niveau. Réalisée en décalage temporel avec

«l'autoconfrontation de premier niveau », mais à partir d'elle, l'autoconfrontation de second niveau prévoit la " participation des acteurs à l'analyse de leur activité ». Au-delà, elle se définit « sur la base de toutes verbalisations provoquées » (Theureau, 2010, p. 312), dans leur deuxième forme, ils s'apparentent à des entretiens ethnographiques (ou de type compréhensif).

Où ?

Sur le lieu de travail. Salle de classe ou bureau de l'acteur.

\section{2 - Illustration d'une succession de signes}

Pour donner un aperçu des pôles et de la concaténation de signes, nous proposons une illustration qui concerne un extrait du « cours d'action » d'un enseignant de $\mathrm{CP}$, lors d'un moment d'activité considéré par lui comme de l'improvisation (voir le tableau, ci-après correspondant au détail des signes 51,52... 61, et 66 du CAS 2, qui mettent entre autres l'accent sur la dynamique d'apprentissage développement). 


\begin{tabular}{|c|c|c|c|c|c|}
\hline $\begin{array}{l}\text { E: } \\
\text { Trouver autre } \\
\text { chose que } \\
\text { «Cerf-volant" } \\
\text { pour } \\
\text { exemplifier } \\
\text { pertinemment } \\
\text { la règle } \\
\text { orthographique. } \\
\text { Attention des } \\
\text { élèves. } \\
\text { Énoncer une } \\
\text { règle } \\
\text { orthographique } \\
\text { «S/sans } S \text { " qui } \\
\text { fonctionne et } \\
\text { qui soit } \\
\text { accessible à des } \\
\text { CP. }\end{array}$ & $\begin{array}{l}\text { A : } \\
\text { «Je " trouvant } \\
\text { versus ne } \\
\text { trouvant pas un } \\
\text { mot-chose qui } \\
\text { convient. } \\
\text { Élèves étant } \\
\text { attentifs. } \\
\text { Élèves } \\
\text { interrompant le } \\
\text { propos versus ne } \\
\text { l'interrompant } \\
\text { pas, intervenant } \\
\text { avec pertinence } \\
\text { versus } \\
\text { impertinence. }\end{array}$ & $\begin{array}{l}\text { S: } \\
\text { [Couper court } \\
\text { aux } \\
\text { interprétations } \\
\text { erronées des } \\
\text { élèves]: est } \\
\text { prioritaire. } \\
\text { [Beaucoup/pas } \\
\text { beaucoup]: } \\
\text { n'est pas } \\
\text { l'argument- } \\
\text { indicateur } \\
\text { pertinent qui } \\
\text { explique la } \\
\text { règle «S/sans } \\
\text { S». } \\
\text { La règle parle } \\
\text { de singulier/ } \\
\text { pluriel. Mais } \\
\text { ces mots ne } \\
\text { doivent pas } \\
\text { dire grand- } \\
\text { chose à des CP. } \\
\text { Connaissance } \\
\text { des mots } \\
\text { "pluriel (qui } \\
\text { dit une quantité } \\
\text { et implique le } \\
\text { «S»àla fin d'un } \\
\text { mot dans le } \\
\text { cadre de la règle } \\
\text { orthographique } \\
\text { correspondante) } \\
\text { et "plusieurs " } \\
\text { (qui dit une } \\
\text { quantité, } \\
\text { davantage } \\
\text { tangible). }\end{array}$ & $\begin{array}{l}\text { R : } \\
\text { Chaîne interprétative } \\
{ }^{20} \text { U50' } \\
\text { Un élève : "Parce que } \\
\text { zéro c'est pas } \\
\text { beaucoup!» (entendu } \\
\text { en arrière-fond } \\
\text { comme subliminal). } \\
\text { " Deux déjà c'est } \\
\text { beaucoup!» }\end{array}$ & 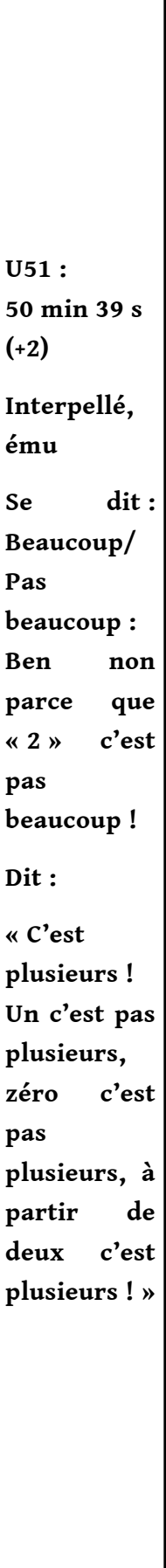 & $\begin{array}{l}\text { I : } \\
\text { [Beaucoup/ } \\
\text { pas } \\
\text { beaucoup]: } \\
\text { ne convient } \\
\text { pas, car } \\
\text { «2» = pas } \\
\text { beaucoup. } \\
\text { [«1 } 1 \text { " et } \\
\text { «0 »]: c'est } \\
\text { pas } \\
\text { plusieurs. } \\
\text { [« } 2 \text { » et >]: } \\
\text { c'est } \\
\text { plusieurs. } \\
\text { [Plusieurs/ } \\
\text { pas } \\
\text { plusieurs]: } \\
\text { peut être le } \\
\text { bon rapport } \\
\text { d'opposition } \\
\text { pour } \\
\text { expliquer la } \\
\text { règle du } \\
\text { pluriel en } \\
\text { CP. }\end{array}$ \\
\hline
\end{tabular}




\begin{tabular}{|c|c|c|c|c|c|}
\hline $\begin{array}{l}\mathrm{E}: \\
\text { Énoncer une } \\
\text { règle } \\
\text { orthographique } \\
\text { «S/sans } \mathrm{S} \text { » qui } \\
\text { soit accessible à } \\
\text { des } \mathrm{CP} \text {. } \\
\text { Attention des } \\
\text { élèves. }\end{array}$ & $\begin{array}{l}\text { A : } \\
\text { Élèves accédant versus } \\
\text { n'accédant pas au sens de la } \\
\text { règle énoncée. } \\
\text { Élèves étant attentifs. } \\
\text { Élèves interrompant le propos } \\
\text { versus ne l'interrompant pas, } \\
\text { intervenant avec pertinence } \\
\text { versus impertinence.. }\end{array}$ & \begin{tabular}{|l} 
S : \\
[Pour faire \\
comprendre \\
un propos] : \\
il r faut \\
utiliser des \\
termes qui \\
restent \\
accessibles \\
aux élèves \\
[Utiliser des \\
mots r \\
inconnus \\
des élèves]: \\
c'est parler \\
dans $\quad$ le \\
vide.
\end{tabular} & $\begin{array}{l}\mathrm{R} \text { : } \\
\text { Chaîne } \\
\text { interprétative U51 }\end{array}$ & $\begin{array}{l} \\
\text { U52: } \\
50 \text { min } 39 \mathrm{~s} \\
(+0) \\
\text { Idée qu'il ne } \\
\text { sait pas si le } \\
\text { mot } \\
\text { "plusieurs = } \\
\text { acquis par les } \\
\text { élèves, leur } \\
\text { parle. }\end{array}$ & $\begin{array}{l}\text { I: } \\
\text { ["plusieurs »]: } \\
\text { n'est pas } \\
\text { nécessairement } \\
\text { un mot qui fait } \\
\text { sens pour des } \\
\text { CP. }\end{array}$ \\
\hline
\end{tabular}

(...) 


\begin{tabular}{|c|c|c|c|c|c|}
\hline 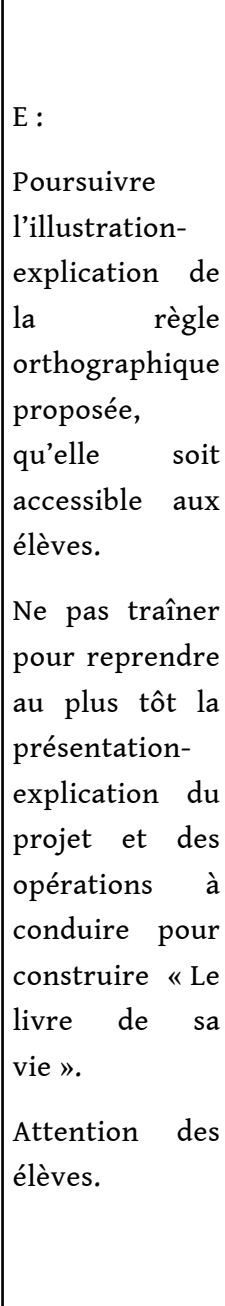 & 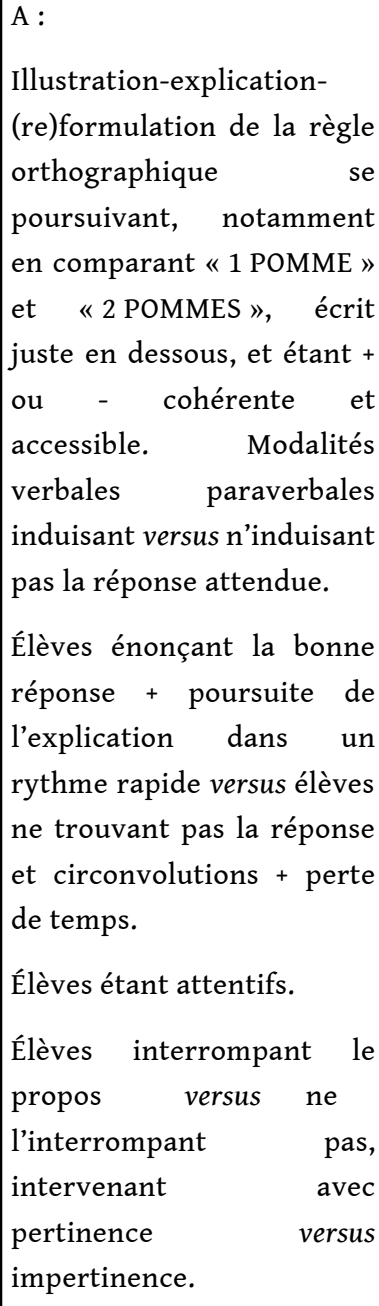 & 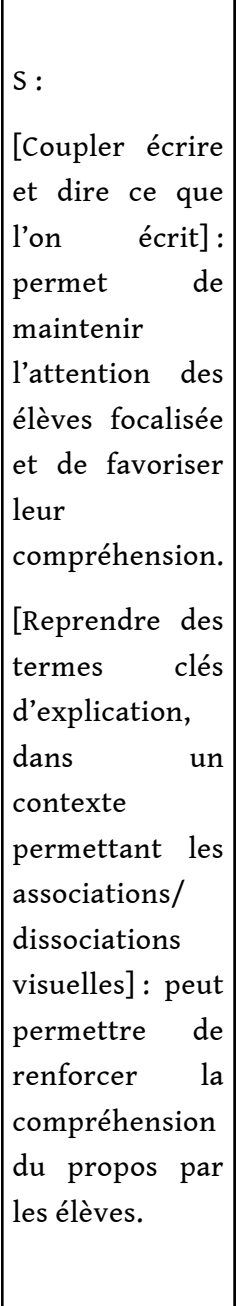 & $\begin{array}{l}\text { R : } \\
\text { Chaîne } \\
\text { interprétative } \\
\text { U60U61 + } \\
\text { Un élève : On } \\
\text { met un «S » à } \\
\text { la fin! } \\
\text { En réponse à } \\
\text { la } \\
\text { question de } \\
\text { l'enseignant : } \\
\text { Si j'écris deux } \\
\text { pommeus } \\
\text { \{Commençant } \\
\text { d'écrire } \\
\text { «2 POMMES » } \\
\text { juste en- } \\
\text { dessous de } \\
\text { «1 POMME »\} } \\
\text { par contre! }\end{array}$ & 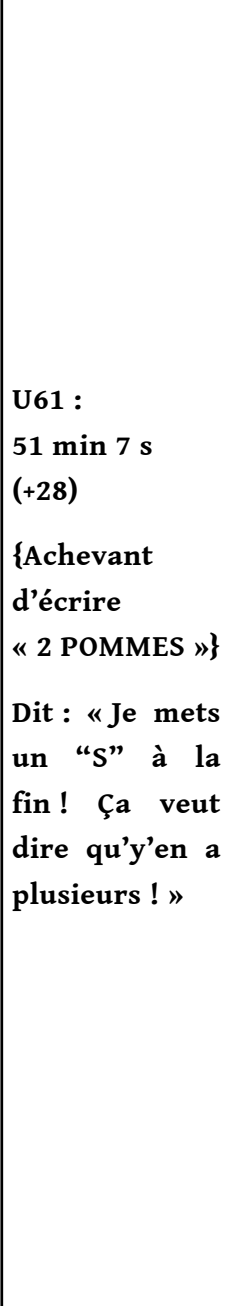 & $\begin{array}{l}\text { I : } \\
\text { [«Plusieurs "]: } \\
\text { est un terme } \\
\text { idoine pour } \\
\text { présenter la } \\
\text { règle } \\
\text { orthographique } \\
\text { (Versus } \\
\text { "pluriel »). }\end{array}$ \\
\hline
\end{tabular}

(...) 


\begin{tabular}{|c|c|c|c|c|c|}
\hline 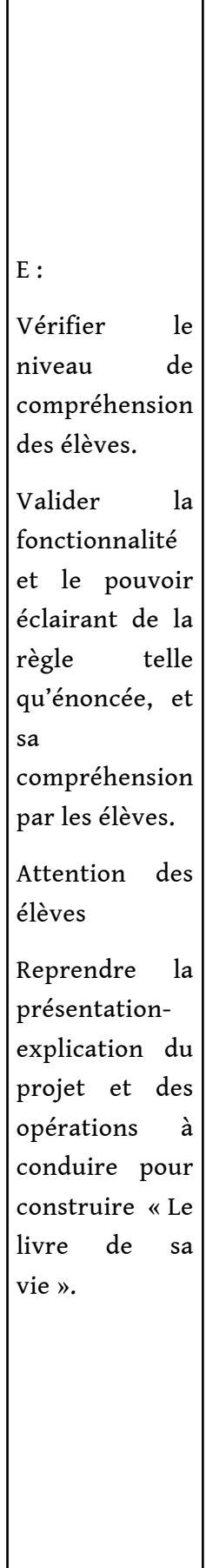 & \begin{tabular}{|l} 
A : \\
Élèves donnant \\
la bonne \\
réponse versus \\
la mauvaise. \\
Volume > \\
d'élèves \\
comprenant- \\
apprenant \\
vraiment versus \\
volume \\
important ne \\
comprenant- \\
apprenant pas.
\end{tabular} & \begin{tabular}{|l} 
S : \\
[Bonne \\
réponse à \\
une \\
question] : \\
signifie que \\
les élèves \\
ont + ou - \\
compris. \\
[Mains qui \\
se lèvent] : \\
sont un \\
indicateur \\
de \\
participation \\
active des \\
élèves.
\end{tabular} & $\begin{array}{l}\text { R: } \\
\text { [Des mains } \\
\text { se lèvent] } \\
\text { Des élèves : } \\
\text { "Mets un } \\
\text { "S"! Oui } \\
\text { mets un } \\
\text { "S"! " la } \\
\text { En réponse } \\
\text { à } \\
\text { question de } \\
\text { l'enseignant } \\
\text { (qui } \\
\text { reprend, } \\
\text { sans trop y } \\
\text { croire, la } \\
\text { proposition } \\
\text { d'un élève) : } \\
\text { Si y'en a } \\
\text { mille! Est-ce } \\
\text { que } \\
\text { quelqu'un } \\
\text { sait? Si y'en } \\
\text { a mille on } \\
\text { met un "S " } \\
\text { ou pas? }\end{array}$ & $\begin{array}{l}\text { U66 : } 51 \text { min } 33 \mathrm{~s} \\
(+26) \\
\text { Sentiment } \\
\text { satisfaction }\end{array}$ & $\begin{array}{l}\text { I: } \\
\text { [Avec cette } \\
\text { classe, } \\
\text { s'embarquer- } \\
\text { digresser un } \\
\text { peu contraint, } \\
\text { en réponse à } \\
\text { une question } \\
\text { assez la } \\
\text { embarrassante } \\
\text { d'un élève]: } \\
\text { peut avoir une } \\
\text { issue positive. } \\
\text { [1000]: fait } \\
\text { suffisamment } \\
\text { sens pour les } \\
\text { élèves. } \\
\text { savent que c'est } \\
\text { plusieurs. } \\
\text { [«Plusieurs »]: } \\
\text { permet, en CP, } \\
\text { une meilleure } \\
\text { appréhension } \\
\text { de la règle. } \\
\text { [Pour une } \\
\text { première } \\
\text { approche de la } \\
\text { règle } \\
\text { orthographique } \\
\text { «S/pas de S » } \\
\text { en cP, la } \\
\text { correspondance } \\
\text { plusieurs/pas } \\
\text { plusieurs]: est } \\
\text { préférable à la } \\
\text { correspondance } \\
\text { singulier/ } \\
\text { pluriel. }\end{array}$ \\
\hline
\end{tabular}

Les pôles ont ici été renseignés à partir de ce que l'acteur a été en mesure de montrer, raconter, commenter de son activité et en prenant en compte les contraintes corporelles, situationnelles et culturelles de son activité. Les données permettant ces analyses sont compilées sur deux supports : (a) un texte synthétique reprenant des données ethnographiques clés, (b) un tableau présentant une description extrinsèque de la situation, ainsi que les verbalisations et actions en interactions en classe et, en correspondance, les verbalisations et actions en entretiens d'autoconfrontation ou de remise en situation dynamique (Theureau, 2010). 


\section{NOTES}

1. Par exemple, en 2010, le référentiel des 10 compétences des maîtres valorisait largement le couple planification/évaluation et les compétences d'anticipation, de prévision, et de régulation qui lui sont associées. Le terme d'improvisation n'est jamais employé. Dans le référentiel 2013 «des compétences professionnelles des métiers du professorat et de l'éducation " (arrêté du 01/07/2013 paru au BO no 30 du 25/07/2013), on note un infléchissement de la place faite au champ lexical de l'anticipation. Les notions les plus proches de celle d'«improvisation " sont celles d'adaptation (en contexte de classe) et d'innovation (sachant que l'innovation est d'abord associée à une activité réflexive hors la classe). Bien sûr on peut comprendre que la notion d'improvisation, dont l'un des sens correspond à un amateurisme douteux, soit tenue à distance par une institution - étant donné le risque de reproche d'incompétence et de discrédit social que représente le fait d'afficher que l'enseignement s'improvise (Perrenoud, 1995).

2. Stagiaires ou titulaires première année, femmes et hommes, spécialistes de disciplines variées et œuvrant dans le premier degré, le second degré ou l'université.

3. Concernant l'utilisation d'artefacts curriculaires, Brown et Edelson (2003) considèrent que l'enseignant adapte lorsqu'il ajuste certains éléments du matériel en apportant des éléments de conception qui lui sont propres mais tout en suivant largement ses consignes; et qu'il improvise lorsque le matériel sert simplement de germe d'idée, d'impulsion pour une bifurcation créatrice radicale en regard des consignes qu'il contient. Pour Piot (2008), en situation imprévue mais dont la structure, la dynamique, l'occurrence ont une proximité avec des situations connues analogues, l'enseignant s'adapte lorsque, sur la base de ses matrices cognitivo-pragmatiques (et éventuellement de leur ajustement mineur), il sort de la routine pour répondre au contexte qu'il rencontre. En situation inédite et jamais rencontrée, l'enseignant improvise (invente) lorsque ses matrices cognitivo-pragmatiques disponibles ne permettent pas de répondre au contexte rencontré.

4. Comme c'est le cas pour certains artistes qui ne considèrent pas que l'improvisation est une simple adaptation, mais une adaptation doublée d'une innovation (e.g. Bailey, 2011), y compris de soi.

5. Chapoulie (1985) rappelle que selon Becker, H., S. (1970). Social Work. Method and substance. New York : Rouledge, la « hiérarchie de crédibilité » conduit le chercheur, s'il n'y prend pas garde, à adopter le point de vue des groupes sociaux les plus légitimes, auxquels, généralement il appartient.

6. L'improvisation étant alors conçue, le concernant, davantage comme l'aptitude à saisir les opportunités interactionnelles au service de l'apprentissage-développement des élèves.

7. La culture est ici conçue comme un ensemble de savoirs, notamment symboliques, partagés par différents acteurs d'une communauté dont l'acteur est lui-même membre (Theureau, 2015). Elle se distingue de la culture propre qui est à comprendre comme l'« ensemble plus ou moins organisé des savoirs symboliques de l'acteur» (Theureau, Ibid., p.51). Et "Comme partie du savoir propre [donc de l'ensemble de savoirs symboliques et non symboliques] d'un acteur» ( Ibid., p. 129). La culture (comme la culture propre ou le savoir propre) étant un corrélat de l'activité située (donc de la dynamique des interactions asymétriques entre un acteur et son environnement), elle dit quelque chose de cette dernière (et vice versa). Activité et culture semblent inextricables et participent de leur dynamique mutuelle.

8. Theureau $(2011,2015)$ qui, depuis 1992, a régulièrement déployé la valeur de l'ethnographie dans le cadre du programme de recherche " cours d'action ", précise qu'elle permet, "par une familiarisation avec les pratiques, la culture et l'histoire personnelle des acteurs» (2011, p. 31) 
ainsi que par le jeu de proximité/distance entre l'ethnographe et les acteurs, en situations, un certain accès à l'asymétrie de leurs couplages respectifs à leur environnement, y compris social.

9. Types d'action, types d'émotions, types de croyances, etc., ou habitudes. On parlera encore de dispositions à agir. Il faut alors les concevoir comme des potentialités plastiques, qui sont à penser à l'interface de l'acteur et de son environnement. Elles sont à appréhender dans un sens peircien, à savoir comme des potentialités qui s'actualisent à des occasions précises (en lien avec un sentiment de familiarité qu'a l'acteur dans le cours de son couplage à son environnement), et comme des tendances qui orientent les comportements (Chauviré, 2002) (ce ne sont pas des déterminations fortes ou des causations mécaniques); mais encore, comme le produit des actions. Si les dispositions possèdent une «disposition à se transformer " (Ibid.), il est aussi à noter que, suivant Peirce, elles peuvent « se fatiguer » (Lahire, 2011).

10. Comme on peut le remarquer, il a d'importantes accointances avec l'anthropologie culturelle, l'anthropologie cognitive, et l'anthropologie sociale ou la sociologie telle que pratiquée par les chercheurs de l'école de Chicago. Theureau $(2004,2006,2015)$ souligne le caractère crucial de la mise en œuvre d'une démarche ethnographique, sur fond de laquelle se déploient les particularités qu'il a jugé pertinent de développer. Extrêmement soucieux du point de vue de l'acteur, en référence à des situations précises d'interaction, sa particularité consiste à avoir cherché à raffiner la prise en compte de son point de vue sur son couplage à son monde, au temps $t$. Et à en renforcer la considération, à partir de développements théoriques et méthodologiques originaux de construction et d'analyse des données.

11. Sachant que pour les 26 occurrences thématiques repérées nous avons un minimum de 0,09/1 concernant l'association improvisation/désinhibition et un maximum de 0,96/1 concernant l'association improvisation/instantanéité. Avec une moyenne générale de 0,61/1 et un écart type de 0,25 .

12. Du fait que nous avons ici opéré par remise en situation dynamique, sans traces vidéographiques et sans avoir observé l'interaction en question.

13. Comprendre Groupe 7 Elève 2.

14. Nous soulignons le fait qu'outre notre passé et notre quotidien de travail en lien avec l'enseignement-apprentissage, nous avons pratiqué le théâtre, la danse contemporaine, le jazz vocal (autant de disciplines artistiques marquées par certaines conceptions et mises en situation ayant, d'une certaine façon, à voir avec l'improvisation).

15. Du grec poiein : « créer, inventer, générer » (voir Luce Girard, In De Certeau, 1990).

16. Soulignons que Rancière (1987, p. 48) entend par là qu'« il n'y a pas de hiérarchie de capacité intellectuelle ». Ce principe d'une égalité « de nature » ne débouche pas sur un égalitarisme mou, autrement dit, il précise qu' «il y a inégalité dans la manifestation de l'intelligence, selon l'énergie plus ou moins grande que la volonté communique à l'intelligence pour découvrir et combiner des rapports nouveaux $»$ (Ibid.).

17. La citation de De Certeau concerne les lecteurs (ou les téléspectateurs). Nous prenons ici le parti de lui donner une portée davantage générique, en parlant d'acteurs.

18. Pour Jullien (2016), la différence et l'écart marquent une séparation, mais la première sous l'angle de la distinction et le second sous celui de la distance. Contrairement à la différence qui, distinction faite, laisse l'un des termes de côté, l'écart suppose de conserver les deux termes, qui restent dépendants l'un de l'autre. «L'écart par la distance entre ouverte entre l'un et l'autre fait apparaître l'“entre" (...) qui n'est ni l'un, ni l'autre, n'a pas d'en soi... » (p. 38-39). L'écart est une ressource. C'est dans/par l'entre, ouvert, que se poursuit, par dérangement, la construction de l'un et de l'autre, sans repli identitaire sur soi, et que se construit le commun (qui n'est pas le semblable).

19. TD pour Travail Dirigé ; CM pour Cours Magistral ; TP pour Travaux Pratiques.

20. Il faut comprendre par chaîne interprétative le fait que le $R$ du signe est contenu dans le $U$ du signe précédent (Theureau, 2004). 


\section{RÉSUMÉS}

Suivant une recherche conduite dans le cadre d'une anthropologie culturelle enactive (Theureau, 2015), cet article aborde le travail des enseignants entrant dans le métier sous l'angle de l'«activité » qui, pour eux, porte le nom d'improvisation. Il propose une autre conception que celle (très répandue) consistant à faire de l'improvisation une sorte de virtuosité interactionnelle d'enseignants experts. Nos résultats, qui permettent notamment de préciser un ensemble de caractéristiques clés, nous conduisent à l'appréhender comme un temps fort de la dynamique du couplage asymétrique entre un néo-enseignant et son environnement (y compris social) et, corrélativement, comme un mouvement majeur d'«individuation» (Simondon, 2013) professionnelle. Cet article propose en outre une discussion concernant le métier d'enseignant, le développement professionnel et la formation à ce métier.

Following research conducted as part of an enactive cultural anthropology (Theureau, 2015), this article examines the work of novice teachers from the perspective of the "activity", which they call improvisation. It proposes a different conception than the (very widespread) one of making improvisation a kind of interactive virtuosity of expert teachers. Our results, which make it possible to specify a set of key characteristics, lead us to consider it as a key moment in the dynamics of asymmetric coupling between a new teacher and his or her environment (including social) and, correlatively, as a major movement of professional "individuation" (Simondon, 2013). This article also provides a discussion about teaching, professional development and teacher education.

\section{INDEX}

Mots-clés : travail enseignant, improvisation, activité, développement professionnel, formation Keywords : teaching, improvisation, activity, professional development, teacher education

\section{AUTEUR}

\section{GUILLAUME AZÉMA}

Université de Montpellier - Laboratoire Interdisciplinaire de Recherche en Didactique, Éducation et Formation (LIRDEF) EA 3749 - guillaume.azema@umontpellier.fr 